\title{
Effects of Si Substitution on the Ei Reaction of
}

\author{
Alkyl Sulfoxides
}

\author{
Supporting Information
}

Ryan McCulla and William S. Jenks*

Department of Chemistry, Iowa State University, Ames IA 50011-3111

\section{Contents}

Table S1: Coordinates and absolute energies $\quad$ S2

Table S2: Calculated heats of isodesmic reactions $\quad$ S33

$\begin{array}{ll}\text { Table S3: Bond lengths, angles, and orders } & \text { S35 }\end{array}$ 
Table S1. Coordinates and Absolute Energies.

\section{(E)-2-methanesulfinylethenylsilane}

$\mathrm{HF} / 6-31 \mathrm{G}(\mathrm{d}, \mathrm{p})$

$\begin{array}{lrrrr}\mathrm{S} & 16.0 & .0114752684 & -.1016740872 & -.8709818060 \\ \mathrm{C} & 6.0 & -.1430368079 & .1154365940 & .9071537675 \\ \mathrm{H} & 1.0 & .3324218808 & 1.0411363315 & 1.2086246650 \\ \mathrm{H} & 1.0 & .3568417339 & -.7200841657 & 1.3792696053 \\ \mathrm{H} & 1.0 & -1.1910668834 & .1147298598 & 1.1763461198 \\ \mathrm{H} & 1.0 & -2.3420674718 & .3028264304 & -2.3010232896 \\ \mathrm{C} & 6.0 & -.8715792630 & 1.3680155668 & -1.3598341515 \\ \mathrm{O} & 8.0 & -.8317331451 & -1.2667516310 & -1.2383872399 \\ \mathrm{H} & 1.0 & -.3917954122 & 2.2911179673 & -1.0760383442 \\ \mathrm{C} & 6.0 & -1.9811749977 & 1.2948118179 & -2.0714852948 \\ \mathrm{SI} & 14.0 & -2.9182210185 & 2.8070185951 & -2.6803608147 \\ \mathrm{H} & 1.0 & -2.2350596712 & 4.0232955359 & -2.1859035187 \\ \mathrm{H} & 1.0 & -4.3083891764 & 2.7810485360 & -2.1747550685 \\ \mathrm{H} & 1.0 & -2.9533413358 & 2.8257551492 & -4.1591891298\end{array}$

Energy

$\mathrm{ZPE}$

MP2 / 6-31G $(\mathrm{d}, \mathrm{p})$

$\begin{array}{lrr}\text { S } & 16.0 & 0.0279525483 \\ \mathrm{C} & 6.0 & -0.1676885408 \\ \mathrm{H} & 1.0 & 0.3059376734 \\ \mathrm{H} & 1.0 & 0.3167209966 \\ \mathrm{H} & 1.0 & -1.2293914947 \\ \mathrm{H} & 1.0 & -2.3002147557 \\ \mathrm{C} & 6.0 & -0.8390768946 \\ \mathrm{O} & 8.0 & -0.8529414747 \\ \mathrm{H} & 1.0 & -0.3581135402 \\ \mathrm{C} & 6.0 & -1.9662677306 \\ \mathrm{SI} & 14.0 & -2.9142308783 \\ \mathrm{H} & 1.0 & -2.2121682867 \\ \mathrm{H} & 1.0 & -4.2930694916 \\ \mathrm{H} & 1.0 & -2.9841744303\end{array}$

Energy

$\mathrm{ZPE}$

MP2 / 6-311+G (3df, 2p)

MP2 / G3Large
-879.4720234183 Hartree

0.107516 HARTREE/MOLECULE
$-0.1248438564$
$-0.8881577958$
0.1367730830
0.8929182382
1.0743710079
1.1799867351
$-0.6922364215$
1.4032334882
0.1507749629
1.1305444862
0.2714990994
1.3763115033
$-2.2955504873$
$-1.3565470439$
$-1.2870762281$
2.3054752286
1.2843558376
$-1.2860428241$
$-1.0675459730$
$-2.0761555663$
2.7885759262
3.9943403096
$-2.6580577756$
$-2.1666843794$
2.7703078756
$-2.1238642001$
$2.8280541718-4.1346414025$

-880.2990192639 Hartree

0.103240 HARTREE/MOLECULE

-880.6678222418 Hartree

-881.3494987954 Hartree 


\section{(z) -2-methanesulfinylethenylsilane}

$\mathrm{HF} / 6-31 \mathrm{G}(\mathrm{d}, \mathrm{p})$

$\begin{array}{lrrrr}\mathrm{S} & 16.0 & 0.1900832233 & -0.0640549234 & -0.6198092609 \\ \mathrm{C} & 6.0 & -0.3703663959 & -0.1852674043 & 1.0834945877 \\ \mathrm{H} & 1.0 & 0.0587996949 & 0.6148690564 & 1.6735792351 \\ \mathrm{H} & 1.0 & -0.0379050484 & -1.1438426374 & 1.4607439338 \\ \mathrm{H} & 1.0 & -1.4519720195 & -0.1462863382 & 1.1119236035 \\ \mathrm{SI} & 14.0 & 1.8382596682 & 3.1051635502 & -1.0763261509 \\ \mathrm{C} & 6.0 & -0.6046314685 & 1.4822147947 & -1.0093111175 \\ \mathrm{O} & 8.0 & -0.5555376876 & -1.1057657529 & -1.3718624641 \\ \mathrm{H} & 1.0 & -1.6698659464 & 1.3648131113 & -1.1408446199 \\ \mathrm{C} & 6.0 & 0.0083776093 & 2.6407323815 & -1.1876325241 \\ \mathrm{H} & 1.0 & -0.6408776327 & 3.4675046837 & -1.4438169061 \\ \mathrm{H} & 1.0 & 2.6850598462 & 1.9754315516 & -0.6541550725 \\ \mathrm{H} & 1.0 & 1.9642243981 & 4.2069729978 & -0.0959865841 \\ \mathrm{H} & 1.0 & 2.2758444587 & 3.5979414290 & -2.3997250601\end{array}$

Energy

$\mathrm{ZPE}$

$\operatorname{MP} 2 / 6-31 G(d, p)$

$\begin{array}{lrr}\mathrm{S} & 16.0 & 0.2230529791 \\ \mathrm{C} & 6.0 & -0.3646553813 \\ \mathrm{H} & 1.0 & 0.0654058211 \\ \mathrm{H} & 1.0 & -0.0407447372 \\ \mathrm{H} & 1.0 & -1.4520471829 \\ \mathrm{SI} & 14.0 & 1.8264228128 \\ \mathrm{C} & 6.0 & -0.6129359325 \\ \mathrm{O} & 8.0 & -0.5062647777 \\ \mathrm{H} & 1.0 & -1.6854440557 \\ \mathrm{C} & 6.0 & -0.0035269636 \\ \mathrm{H} & 1.0 & -0.6665557026 \\ \mathrm{H} & 1.0 & 2.6413414526 \\ \mathrm{H} & 1.0 & 1.9872124299 \\ \mathrm{H} & 1.0 & 2.2782319377\end{array}$

Energy

$\mathrm{ZPE}$

MP2 / 6-311+G (3df, 2p)
-879.4642809539 Hartree

0.107068 HARTREE/MOLECULE
$-0.0698983342-0.6368901288$

$-0.1591921343 \quad 1.0727132682$

$0.6567341553 \quad 1.6511302745$

$-1.1163787281 \quad 1.4759239210$

$-0.1098887599 \quad 1.0846927504$

$3.0916386290-1.0707083345$

$1.4756746446-0.9896676879$

$-1.1400154098-1.4167730293$

$1.3372247117-1.1119656197$

$2.6589089406-1.1773404494$

$3.4866554432-1.4190349265$

$1.9356387436-0.6571756504$

$4.1823434817-0.0843369641$

$3.5809811167-2.3902958235$

-880.2904084985 Hartree

0.102829 HARTREE/MOLECULE

-880.6605017582 Hartree 


\section{1-methanesulfinylethenylsilane}

$\mathrm{HF} / 6-31 \mathrm{G}(\mathrm{d}, \mathrm{p})$

$\begin{array}{lrrrr}\mathrm{S} & 16.0 & .0245167551 & -.0097903577 & -.9941486058 \\ \mathrm{C} & 6.0 & .0577022419 & -.0286559787 & .8030085933 \\ \mathrm{H} & 1.0 & .5687455927 & .8465541492 & 1.1835404452 \\ \mathrm{H} & 1.0 & .5940034478 & -.9206970698 & 1.0991126409 \\ \mathrm{H} & 1.0 & -.9573534634 & -.0734341398 & 1.1752353802 \\ \mathrm{H} & 1.0 & -2.5347584205 & . .3291150802 & -1.9669094468 \\ \mathrm{C} & 6.0 & -.9539217324 & 1.4784734646 & -1.2221913881 \\ \mathrm{O} & 8.0 & -.8136288483 & -1.1684540452 & -1.3969430461 \\ \mathrm{SI} & 14.0 & -.2113746511 & 3.1856934834 & -.9124856563 \\ \mathrm{C} & 6.0 & -2.1614150916 & 1.3133057427 & -1.7404844415 \\ \mathrm{H} & 1.0 & -2.7989265107 & 2.1533937331 & -1.9556913387 \\ \mathrm{H} & 1.0 & .1334036662 & 3.3667182447 & .5154358596 \\ \mathrm{H} & 1.0 & -1.2212751366 & 4.1875607143 & -1.3087300286 \\ \mathrm{H} & 1.0 & 1.0228821509 & 3.3579369791 & -1.7078089673\end{array}$

Energy

$\mathrm{ZPE}$

$\operatorname{MP} 2 / 6-31 G(d, p)$

$\begin{array}{lrr}\mathrm{S} & 16.0 & .0305909313 \\ \mathrm{C} & 6.0 & .0539351851 \\ \mathrm{H} & 1.0 & .5561056814 \\ \mathrm{H} & 1.0 & .5940149999 \\ \mathrm{H} & 1.0 & -.9729235025 \\ \mathrm{H} & 1.0 & -2.5161817787 \\ \mathrm{C} & 6.0 & -.9411663986 \\ \mathrm{O} & 8.0 & -.8432357226 \\ \mathrm{SI} & 14.0 & -.1983760414 \\ \mathrm{C} & 6.0 & -2.1657969662 \\ \mathrm{H} & 1.0 & -2.8220957213 \\ \mathrm{H} & 1.0 & .1434274901 \\ \mathrm{H} & 1.0 & -1.2064025877 \\ \mathrm{H} & 1.0 & 1.0367044313\end{array}$

Energy

$\mathrm{ZPE}$

MP2 / 6-311+G (3df, 2p)
$-.0343339671$

$-1.0233448711$

$-.0000551423$

.8972598246

.7851842286

$-.8831623028$

1.1411931705

$-.0364127323$

1.1184435203

.3011067533

1.1434908529

$-1.9642771903$

1.4801641245

$-1.2019659619$

3.1771610183

1.3021443353

2.1380952274

3.3476038865

$-1.2113152445$

$-1.4234711716$

$-.8908888776$

$-1.7345687863$

$-1.9366921924$

.5379778631

$4.1807330911-1.2867138301$

$3.3493818454-1.6840774713$
-880.3000882174 Hartree .103326 HARTREE/MOLECULE -880.6694030298 Hartree 


\section{(E)-2-methanesulfinylethenylsilane TS (H-transfer)}

$\mathrm{HF} / 6-31 \mathrm{G}(\mathrm{d}, \mathrm{p})$

$\begin{array}{lrrrr}\mathrm{S} & 16.0 & 1.2107399681 & -1.1916475922 & .5333347057 \\ \mathrm{C} & 6.0 & .9108350487 & -.9547301340 & 2.2849437102 \\ \mathrm{H} & 1.0 & -.0281054087 & -.4350749799 & 2.4158431477 \\ \mathrm{H} & 1.0 & 1.7250664322 & -.3601068774 & 2.6835938293 \\ \mathrm{H} & 1.0 & .8870469732 & -1.9154900128 & 2.7807953286 \\ \mathrm{C} & 6.0 & .6862992577 & .6533965774 & -.1693220487 \\ \mathrm{O} & 8.0 & -.1271739487 & -1.8734366830 & .0472332179 \\ \mathrm{H} & 1.0 & -.6613445863 & -1.0868476547 & -.0312243252 \\ \mathrm{H} & 1.0 & 1.5333069386 & 1.2860135471 & -.0006347072 \\ \mathrm{C} & 6.0 & -.4925727800 & .5987552986 & -.6479939991 \\ \mathrm{SI} & 14.0 & -1.5160696032 & 1.9321358027 & -1.4065948391 \\ \mathrm{H} & 1.0 & -.8458049788 & 3.2585076586 & -1.4793742865 \\ \mathrm{H} & 1.0 & -2.7559759351 & 2.0997600197 & -.6125563045 \\ \mathrm{H} & 1.0 & -1.8908073777 & 1.5403650298 & -2.7846834292\end{array}$

Energy

$\mathrm{ZPE}$

-879.4041784888 Hartree

0.103789 HARTREE/MOLECULE

$\operatorname{MP} 2 / 6-31 G(d, p)$

$\begin{array}{lrrrr}\mathrm{S} & 16.0 & 1.1596890557 & -1.3773173215 & .5258126272 \\ \mathrm{C} & 6.0 & .8681049050 & -.9931498432 & 2.2608657925 \\ \mathrm{H} & 1.0 & .0735012628 & -.2545127697 & 2.3308457838 \\ \mathrm{H} & 1.0 & 1.7889158189 & -.5857165736 & 2.6782741261 \\ \mathrm{H} & 1.0 & .5912976794 & -1.8953049928 & 2.7995887537 \\ \mathrm{C} & 6.0 & .9033952272 & .7460136108 & -.1367654368 \\ \mathrm{O} & 8.0 & -.2980209502 & -1.6637047075 & -.0315020339 \\ \mathrm{H} & 1.0 & -.6005949715 & -.6086470595 & -.4162905275 \\ \mathrm{H} & 1.0 & 1.8117481534 & 1.2730768356 & .0879961720 \\ \mathrm{C} & 6.0 & -.2720494451 & .7698899861 & -.6162412985 \\ \mathrm{SI} & 14.0 & -1.5698962223 & 1.8241832569 & -1.3836419322 \\ \mathrm{H} & 1.0 & -1.1166571889 & 3.2232197449 & -1.5496546525 \\ \mathrm{H} & 1.0 & -2.7749608034 & 1.8148454293 & -.5264539304 \\ \mathrm{H} & 1.0 & -1.9290325211 & 1.2787244041 & -2.7094734433\end{array}$

Energy

$\mathrm{ZPE}$

$\mathrm{MP} 2 / 6-311+\mathrm{G}(3 \mathrm{df}, 2 \mathrm{p})$

MP2/G3-large
-880.2481329653 Hartree .097027 HARTREE/MOLECULE

-880.6126446114 Hartree

-881.2940094489 Hartree 


\section{(z) -2-methanesulfinylethenylsilane TS (Si-transfer)}

$\mathrm{HF} / 6-31 \mathrm{G}(\mathrm{d}, \mathrm{p})$

$\begin{array}{lrrrr}\mathrm{S} & 16.0 & 0.9079717591 & -0.9225359607 & 0.3170357391 \\ \mathrm{C} & 6.0 & 0.4662092956 & -0.8374875145 & 2.0518721934 \\ \mathrm{H} & 1.0 & -0.0940835994 & 0.0676559669 & 2.2439849176 \\ \mathrm{H} & 1.0 & 1.3888022366 & -0.8259679374 & 2.6216739032 \\ \mathrm{H} & 1.0 & -0.1152916135 & -1.7077118414 & 2.3241635961 \\ \mathrm{C} & 6.0 & 1.3573358417 & 1.2816393236 & -0.0528992520 \\ \mathrm{O} & 8.0 & -0.5273212545 & -0.9284736527 & -0.3666102006 \\ \mathrm{SI} & 14.0 & -1.6259770011 & 0.2543690155 & -0.9763418659 \\ \mathrm{H} & 1.0 & 2.3739710843 & 1.2663954343 & 0.2534437097 \\ \mathrm{C} & 6.0 & 0.2941765252 & 1.7511088402 & -0.5012424008 \\ \mathrm{H} & 1.0 & 0.0474267594 & 2.7512827895 & -0.7946277086 \\ \mathrm{H} & 1.0 & -1.3815714486 & 0.8364867291 & -2.3116499171 \\ \mathrm{H} & 1.0 & -2.2688458714 & 1.1658442312 & -0.0052946525 \\ \mathrm{H} & 1.0 & -2.6913755134 & -0.7564915237 & -1.2450237616\end{array}$

Energy

$\mathrm{ZPE}$

$\operatorname{MP} 2 / 6-31 G(d, p)$

$\begin{array}{lrr}\mathrm{S} & 16.0 & 0.2230529791 \\ \mathrm{C} & 6.0 & -0.3646553813 \\ \mathrm{H} & 1.0 & 0.0654058211 \\ \mathrm{H} & 1.0 & -0.0407447372 \\ \mathrm{H} & 1.0 & -1.4520471829 \\ \mathrm{SI} & 14.0 & 1.8264228128 \\ \mathrm{C} & 6.0 & -0.6129359325 \\ \mathrm{O} & 8.0 & -0.5062647777 \\ \mathrm{H} & 1.0 & -1.6854440557 \\ \mathrm{C} & 6.0 & -0.0035269636 \\ \mathrm{H} & 1.0 & -0.6665557026 \\ \mathrm{H} & 1.0 & 2.6413414526 \\ \mathrm{H} & 1.0 & 1.9872124299 \\ \mathrm{H} & 1.0 & 2.2782319377\end{array}$

Energy

$\mathrm{ZPE}$

$\operatorname{MP} 2 / 6-311+G(3 d f, 2 p)$
-879.4026929260 Hartree

0.103230 HARTREE/MOLECULE

$\begin{array}{rr}-0.0698983342 & -0.6368901288 \\ -0.1591921343 & 1.0727132682 \\ 0.6567341553 & 1.6511302745 \\ -1.1163787281 & 1.4759239210 \\ -0.1098887599 & 1.0846927504 \\ 3.0916386290 & -1.0707083345 \\ 1.4756746446 & -0.9896676879 \\ -1.1400154098 & -1.4167730293 \\ 1.3372247117 & -1.1119656197 \\ 2.6589089406 & -1.1773404494 \\ 3.4866554432 & -1.4190349265 \\ 1.9356387436 & -0.6571756504 \\ 4.1823434817 & -0.0843369641 \\ 3.5809811167 & -2.3902958235\end{array}$

-880.2386200349 Hartree 0.103219 HARTREE/MOLECULE -880.6031018145 Hartree 


\section{1-methanesulfinylethenylsilane TS (H-transfer)}

$\mathrm{HF} / 6-31 \mathrm{G}(\mathrm{d}, \mathrm{p})$

$\begin{array}{lrrrr}\mathrm{S} & 16.0 & -.3628804461 & -1.4747965549 & -.3078393056 \\ \mathrm{C} & 6.0 & -.9821562030 & -1.6157997250 & 1.3695398413 \\ \mathrm{H} & 1.0 & -1.8204812412 & -.9450369603 & 1.4975502715 \\ \mathrm{H} & 1.0 & -.1823160776 & -1.3469591652 & 2.0491126091 \\ \mathrm{H} & 1.0 & -1.2861131688 & -2.6375419847 & 1.5524807902 \\ \mathrm{C} & 6.0 & -.5199319816 & .6595818070 & -.5752481506 \\ \mathrm{O} & 8.0 & -1.6553993978 & -1.7566754453 & -1.1642852814 \\ \mathrm{H} & 1.0 & -2.0047703241 & -.7940997355 & -1.3744884353 \\ \mathrm{SI} & 14.0 & 1.0000602589 & 1.4946944731 & .0862968018 \\ \mathrm{C} & 6.0 & -1.6260372103 & .7857793725 & -1.2054263472 \\ \mathrm{H} & 1.0 & -2.0393129600 & 1.7340827959 & -1.5017029326 \\ \mathrm{H} & 1.0 & 2.1898262130 & 1.1231009430 & -.7090578114 \\ \mathrm{H} & 1.0 & 1.2439133297 & 1.0757236949 & 1.4864695581 \\ \mathrm{H} & 1.0 & .7861786090 & 2.9550018845 & .0306247922\end{array}$

Energy

$\mathrm{ZPE}$

$\operatorname{MP} 2 / 6-31 G(d, p)$

$\begin{array}{lrr}\mathrm{S} & 16.0 & -.3407048432 \\ \mathrm{C} & 6.0 & -.9228890173 \\ \mathrm{H} & 1.0 & -1.6483426249 \\ \mathrm{H} & 1.0 & -.0682610177 \\ \mathrm{H} & 1.0 & -1.3783074438 \\ \mathrm{C} & 6.0 & -.5221420970 \\ \mathrm{O} & 8.0 & -1.6830643231 \\ \mathrm{H} & 1.0 & -1.9370943632 \\ \mathrm{SI} & 14.0 & .9914150594 \\ \mathrm{C} & 6.0 & -1.6257867487 \\ \mathrm{H} & 1.0 & -2.3507615935 \\ \mathrm{H} & 1.0 & 2.1680975140 \\ \mathrm{H} & 1.0 & 1.1488965069 \\ \mathrm{H} & 1.0 & .9095243922\end{array}$

Energy

$\mathrm{ZPE}$

$\operatorname{MP} 2 / 6-311+G(3 d f, 2 p)$
-879.3945157441 Hartree .102071 HARTREE/MOLECULE
$-.3507647274$

1.3533038297

1.4670401499

2.0040865569

1.6031150234

$-.5984477609$

$-1.1758853345$

$-1.3661013646$

.1365420017

$-1.2221344425$

$-1.6450149772$

$-.6498796373$

1.5217745094

.1563925737

.9961180326

2.9685710696

-880.2437871827 Hartree

0.096443 HARTREE/MOLECULE

-880.6081467952 Hartree 


\section{Silyl methanesulfenate}

$\mathrm{HF} / 6-31 \mathrm{G}(\mathrm{d}, \mathrm{p})$

\begin{tabular}{|c|c|c|c|c|}
\hline S & 16.0 & -.0721827537 & 1.1392803557 & .0892813104 \\
\hline C & 6.0 & -1.5629122837 & .6077490780 & .9364234745 \\
\hline $\mathrm{H}$ & 1.0 & -1.3087186185 & .0570202470 & 1.8318353443 \\
\hline $\mathrm{H}$ & 1.0 & -2.1893123153 & .0047674864 & .2926301265 \\
\hline $\mathrm{H}$ & 1.0 & -2.0987559408 & 1.5097874211 & 1.2111736166 \\
\hline O & 8.0 & .6370001322 & -.3396585042 & -.1678238651 \\
\hline SI & 14.0 & .5927008603 & -1.0589164799 & -1.6708890669 \\
\hline $\mathrm{H}$ & 1.0 & 1.2795460258 & -2.3550334379 & -1.5267521660 \\
\hline $\mathrm{H}$ & 1.0 & 1.2842041614 & -.2200221560 & -2.670558183 \\
\hline $\mathrm{H}$ & 1.0 & -.8076131677 & -1.2669495104 & -2.099338890 \\
\hline $\begin{array}{l}\text { Energy } \\
\text { ZPE }\end{array}$ & & & $\begin{array}{l}-802.6550636965 \\
.072652 \text { HARTREE }\end{array}$ & $\begin{array}{l}\text { Hart } \\
\text { MOLI }\end{array}$ \\
\hline
\end{tabular}

$\operatorname{MP} 2 / 6-31 G(d, p)$

$\begin{array}{lrrrr}\mathrm{S} & 16.0 & -.0433148266 & 1.1455804711 & .0861129428 \\ \mathrm{C} & 6.0 & -1.5439163356 & .5888753486 & .8938223281 \\ \mathrm{H} & 1.0 & -1.3033603319 & .0744978108 & 1.8204445438 \\ \mathrm{H} & 1.0 & -2.1290145404 & -.0587974569 & .2456544262 \\ \mathrm{H} & 1.0 & -2.1263687021 & 1.4814245557 & 1.1223764001 \\ \mathrm{O} & 8.0 & .7201603368 & -.3693206937 & -.0876776203 \\ \mathrm{SI} & 14.0 & .5698754220 & -1.0372523834 & -1.6409046881 \\ \mathrm{H} & 1.0 & 1.2302739017 & -2.3524176613 & -1.5554271712 \\ \mathrm{H} & 1.0 & 1.2344285767 & -.1945663456 & -2.6551400832 \\ \mathrm{H} & 1.0 & -.8548074008 & -1.1999991453 & -2.0032793782 \\ & & & -803.2073476404 & \text { Hartree } \\ \text { Energy } & & & 0.070377 \text { HARTREE/MOLECULE } \\ \text { ZPE } & & & -803.4844863319 & \text { Hartree } \\ \text { MP2/6-311+G(3df,2p) } & & \end{array}$




\section{Ethynylsilane}

$\mathrm{HF} / 6-31 \mathrm{G}(\mathrm{d}, \mathrm{p})$

$\begin{array}{lrrrr}\mathrm{H} & 1.0 & .0508282320 & .0050566194 & -3.0244603491 \\ \mathrm{C} & 6.0 & .0330894913 & .0014715873 & -1.9665342292 \\ \mathrm{C} & 6.0 & .0130949766 & -.0016740538 & -.07728092250 \\ \mathrm{SI} & 14.0 & -.0181660243 & .0005619279 & 1.0677613240 \\ \mathrm{H} & 1.0 & 1.1768135825 & -.0994537195 & 1.5801549317 \\ \mathrm{H} & 1.0 & -1.2353049843 & -.0900863334 & 1.5390730083 \\ \mathrm{H} & 1.0 & -.0209832736 & 1.3946917721 & 1.5544295392\end{array}$

Energy

$\mathrm{ZPE}$

-366.9204338961 Hartree .046825 HARTREE/MOLECULE

$\operatorname{MP} 2 / 6-31 G(d, p)$

$\begin{array}{lrrrr}\mathrm{H} & 1.0 & .0513164498 & .0040557733 & -3.0509771069 \\ \mathrm{C} & 6.0 & .0334338188 & .0017869180 & -1.9867992004 \\ \mathrm{C} & 6.0 & .0127993101 & -.0010489978 & -.0584470344 \\ \mathrm{SI} & 14.0 & -.0182495663 & .0007988578 & 1.0729761819 \\ \mathrm{H} & 1.0 & 1.1751120567 & -.06985896055 & 1.5886690562 \\ \mathrm{H} & 1.0 & -1.2338634801 & -.6892028422 & 1.5477106832 \\ \mathrm{H} & 1.0 & -.0211765889 & 1.3927676964 & 1.5644824202\end{array}$

Energy

$\mathrm{ZPE}$

MP2 / 6-311+G (3df, 2p)

MP2 / G3Large
-367.2713793588 Hartree .043984 HARTREE/MOLECULE -367.4026503109 Hartree -367.7283468001 Hartree 


\section{(E) -methanesulfinylpropene}

$\mathrm{HF} / 6-31 \mathrm{G}(\mathrm{d}, \mathrm{p})$

$\begin{array}{rrrrr}\mathrm{S} & 16.0 & -.0536522338 & -.1558389921 & -.8919769250 \\ \mathrm{C} & 6.0 & -.0884495999 & .1345718118 & .8813243982 \\ \mathrm{H} & 1.0 & .4673329402 & 1.0339302347 & 1.1186578579 \\ \mathrm{H} & 1.0 & .3770401088 & -.7174536588 & 1.3596365348 \\ \mathrm{H} & 1.0 & -1.1169791656 & .2220378181 & 1.2060739642 \\ \mathrm{H} & 1.0 & -2.4788077225 & .3489859392 & -2.1701175559 \\ \mathrm{C} & 6.0 & -.8577142575 & 1.3439923312 & -1.4001991624 \\ \mathrm{O} & 8.0 & -.9943815920 & -1.2762723617 & -1.1565513192 \\ \mathrm{H} & 1.0 & -.2994790760 & 2.2479078815 & -1.2193821535 \\ \mathrm{C} & 6.0 & -2.0193043718 & 1.3123089170 & -2.0210470077 \\ \mathrm{C} & 6.0 & -2.7479454614 & 2.5161230442 & -2.5400552894 \\ \mathrm{H} & 1.0 & -2.2087605061 & 3.4344618164 & -2.3395281606 \\ \mathrm{H} & 1.0 & -3.7315372256 & 2.5876819519 & -2.0845015310 \\ \mathrm{H} & 1.0 & -2.8990674365 & 2.4325856667 & -3.6126301507\end{array}$

Energy

$\mathrm{ZPE}$

$\operatorname{MP} 2 / 6-31 G(d, p)$

$\begin{array}{lrr}\mathrm{S} & 16.0 & -.0541530790 \\ \mathrm{C} & 6.0 & -.0941345928 \\ \mathrm{H} & 1.0 & .4595134858 \\ \mathrm{H} & 1.0 & .3715933109 \\ \mathrm{H} & 1.0 & -1.1320869668 \\ \mathrm{H} & 1.0 & -2.4507368893 \\ \mathrm{C} & 6.0 & -.8381518322 \\ \mathrm{O} & 8.0 & -1.0368252084 \\ \mathrm{H} & 1.0 & -.2786536597 \\ \mathrm{C} & 6.0 & -2.0102869159 \\ \mathrm{C} & 6.0 & -2.7468551300 \\ \mathrm{H} & 1.0 & -2.2052258374 \\ \mathrm{H} & 1.0 & -3.7323772286 \\ \mathrm{H} & 1.0 & -2.9033250562\end{array}$

Energy

$\mathrm{ZPE}$

MP2 / 6-311+G (3df, 2p)
-628.4333717171 Hartree .121127 HARTREE/MOLECULE

$\begin{array}{rr}-.1848707137 & -.9074100516 \\ .1495544560 & .8705870309 \\ 1.0621208376 & 1.0855197997 \\ -.6930362052 & 1.3764573486 \\ .2448632005 & 1.1828123879 \\ .3274808897 & -2.1831982820 \\ 1.3474496193 & -1.3858494749 \\ -1.3028007420 & -1.1808276792 \\ 2.2552074347 & -1.1875738927 \\ 1.3076570585 & -2.0260150860 \\ 2.5084196242 & -2.5279403974 \\ 3.4288350761 & -2.3163870940 \\ 2.5741817527 & -2.0653563118 \\ 2.4399601116 & -3.6051147979\end{array}$

-629.3105656559Hartree .116478 HARTREE/MOLECULE -629.6641946730 Hartree 


\section{(Z) -methanesulfinylpropene}

$\mathrm{HF} / 6-31 \mathrm{G}(\mathrm{d}, \mathrm{p})$

$\begin{array}{lrrrr}\mathrm{C} & 6.0 & -0.3299656921 & 0.5698003330 & -0.5484258078 \\ \mathrm{C} & 6.0 & 0.3624778957 & 0.1293751792 & -1.8063210487 \\ \mathrm{H} & 1.0 & 0.6223109461 & 1.0052779469 & -2.3959120441 \\ \mathrm{H} & 1.0 & -0.2443462114 & -0.5321089138 & -2.4013496283 \\ \mathrm{H} & 1.0 & 1.2957982879 & -0.3645513134 & -1.5487397821 \\ \mathrm{C} & 6.0 & -1.5417573588 & 0.2898375288 & -0.1015600666 \\ \mathrm{~S} & 16.0 & -2.7690665636 & -0.7943487907 & -0.7934961368 \\ \mathrm{C} & 6.0 & -4.0225467751 & 0.4559581661 & -1.1073333257 \\ \mathrm{H} & 1.0 & -4.8825823961 & -0.0516293984 & -1.5245372458 \\ \mathrm{H} & 1.0 & -3.6390061115 & 1.1735075261 & -1.8207185934 \\ \mathrm{H} & 1.0 & -4.3007406598 & 0.9452562868 & -0.1819009791 \\ \mathrm{O} & 8.0 & -2.3423892981 & -1.2966295069 & -2.1257827936 \\ \mathrm{H} & 1.0 & -1.8690396400 & 0.6863808232 & 0.8463918344 \\ \mathrm{H} & 1.0 & 0.2549654168 & 1.2170236251 & 0.0886476525\end{array}$

Energy

$\mathrm{ZPE}$

$\operatorname{MP} 2 / 6-31 G(d, p)$

$\begin{array}{lrr}\mathrm{C} & 6.0 & -0.3255803480 \\ \mathrm{C} & 6.0 & 0.3549393213 \\ \mathrm{H} & 1.0 & 0.6092213698 \\ \mathrm{H} & 1.0 & -0.2703834660 \\ \mathrm{H} & 1.0 & 1.2932645827 \\ \mathrm{C} & 6.0 & -1.5596434621 \\ \mathrm{~S} & 16.0 & -2.7581166522 \\ \mathrm{C} & 6.0 & -4.0080919868 \\ \mathrm{H} & 1.0 & -4.8875525412 \\ \mathrm{H} & 1.0 & -3.6171978812 \\ \mathrm{H} & 1.0 & -4.2600529964 \\ \mathrm{O} & 8.0 & -2.3223541332 \\ \mathrm{H} & 1.0 & -1.9096046399 \\ \mathrm{H} & 1.0 & 0.2552646731\end{array}$

Energy

$\mathrm{ZPE}$

MP2 / 6-311+G (3df, 2p)
-628.4278554058 Hartree

0.121340 HARTREE/MOLECULE

$\begin{array}{rr}0.5858117425 & -0.5444050186 \\ 0.1291609159 & -1.7982851273 \\ 0.9944720571 & -2.4126151348 \\ -0.5435824303 & -2.3735505504 \\ -0.3667657043 & -1.5461217931 \\ 0.2958926394 & -0.1058663321 \\ -0.8219073469 & -0.8172325584 \\ 0.4574067167 & -1.0991693700 \\ -0.0351418071 & -1.5074503082 \\ 1.1754875964 & -1.8168840187 \\ 0.9469176265 & -0.1603979995 \\ -1.3190877424 & -2.1786808296 \\ 0.6907886626 & 0.8430120284 \\ 1.2436965659 & 0.0966090472\end{array}$

-629.3056277977 Hartree 0.116313 HARTREE/MOLECULE -629.6586586490 Hartree 


\section{2-methanesulfinylpropene}

$\mathrm{HF} / 6-31 \mathrm{G}(\mathrm{d}, \mathrm{p})$

$\begin{array}{rrrrr}\mathrm{S} & 16.0 & .0610677465 & .0000882178 & -.8401603218 \\ \mathrm{C} & 6.0 & -.1684459834 & .0986368346 & .9405924924 \\ \mathrm{H} & 1.0 & .2969004511 & .9921901634 & 1.3361091166 \\ \mathrm{H} & 1.0 & .3084805668 & -.7750680508 & 1.3650654537 \\ \mathrm{H} & 1.0 & -1.2269149847 & .0805962284 & 1.1653260616 \\ \mathrm{H} & 1.0 & -2.3304893977 & .2624715031 & -2.2200210989 \\ \mathrm{C} & 6.0 & -.8829858804 & 1.4509145479 & -1.3140970530 \\ \mathrm{O} & 8.0 & -.6979377852 & -1.1977816609 & -1.2827342487 \\ \mathrm{C} & 6.0 & -.2795279887 & 2.7869859534 & -.9682866292 \\ \mathrm{C} & 6.0 & -1.9898260023 & 1.2571725675 & -2.0005001420 \\ \mathrm{H} & 1.0 & -2.5699989390 & 2.0893377911 & -2.3600688730 \\ \mathrm{H} & 1.0 & .7604015183 & 2.8391015550 & -1.2771761882 \\ \mathrm{H} & 1.0 & -.3154666071 & 2.9836501087 & .0990151412 \\ \mathrm{H} & 1.0 & -.8206756142 & 3.5792646409 & -1.4706765108\end{array}$

Energy

$\mathrm{ZPE}$

MP2 / 6-31G (d, p)

$\begin{array}{lrr}\mathrm{S} & 16.0 & 0.0680685845 \\ \mathrm{C} & 6.0 & -0.1566155101 \\ \mathrm{H} & 1.0 & 0.2972368912 \\ \mathrm{H} & 1.0 & 0.3317442598 \\ \mathrm{H} & 1.0 & -1.2226082878 \\ \mathrm{H} & 1.0 & -2.3279161485 \\ \mathrm{C} & 6.0 & -0.8754016656 \\ \mathrm{O} & 8.0 & -0.7316347474 \\ \mathrm{C} & 6.0 & -0.2731372605 \\ \mathrm{C} & 6.0 & -2.0056350264 \\ \mathrm{H} & 1.0 & -2.6065255112 \\ \mathrm{H} & 1.0 & 0.7937796867 \\ \mathrm{H} & 1.0 & -0.3918275133 \\ \mathrm{H} & 1.0 & -0.7549466512 \\ \mathrm{H} & 1.0 & 0.2552646731\end{array}$

Energy

$\mathrm{ZPE}$

MP2 / 6-311+G (3df, 2p)
$-0.0283322135$

0.1264121298

1.0464915772

$-0.7291447393$

0.1050232622

0.2431023402

1. 4506500817

$-1.2342066796$

2.7737432568

1.2516379102

2.0835142557

2.7955093847

2.9927296836

3.5704301503

1.2436965659
$-0.8771400064$

0.9124509904

1.2747259760

1.3727680026

1.1306019030

$-2.2128000748$

$-1.3058558222$

$-1.3168666257$

$-0.9387196522$

$-1.9905964739$

$-2.3317154615$

$-1.1647155550$

0.1228253025

$-1.5025753029$

0.0966090472

-629.3116314154 Hartree 0.115965 HARTREE/MOLECULE -629.6660595503 Hartree 


\section{2-methanesulfinylpropene TS (H-Transfer)}

$\mathrm{HF} / 6-31 \mathrm{G}(\mathrm{d}, \mathrm{p})$

$\begin{array}{rrrrr}\mathrm{S} & 16.0 & -.2655812263 & -1.3885226591 & -.3150314500 \\ \mathrm{C} & 6.0 & -.8645739794 & -1.5645822808 & 1.3674530946 \\ \mathrm{H} & 1.0 & -1.6861771482 & -.8801819471 & 1.5285280400 \\ \mathrm{H} & 1.0 & -.0490464661 & -1.3360196920 & 2.0437209514 \\ \mathrm{H} & 1.0 & -1.1886557178 & -2.5838979740 & 1.5274415900 \\ \mathrm{C} & 6.0 & -.4181560879 & .7529798997 & -.5423251410 \\ \mathrm{O} & 8.0 & -1.5717520334 & -1.6521232200 & -1.1620421740 \\ \mathrm{H} & 1.0 & -1.9064393295 & -.6865888587 & -1.3529942821 \\ \mathrm{C} & 6.0 & .8035795411 & 1.3358122516 & .0731989660 \\ \mathrm{C} & 6.0 & -1.5120184627 & .9055737666 & -1.1610962192 \\ \mathrm{H} & 1.0 & -1.9393531495 & 1.8469619788 & -1.4533974379 \\ \mathrm{H} & 1.0 & 1.7065148566 & .9742830694 & -.04075984810 \\ \mathrm{H} & 1.0 & .8715647294 & 1.1203780992 & 1.1354429051 \\ \mathrm{H} & 1.0 & .7606738735 & 2.4129829663 & -.0472739620\end{array}$

Energy

$\mathrm{ZPE}$

$\operatorname{MP} 2 / 6-31 G(d, p)$

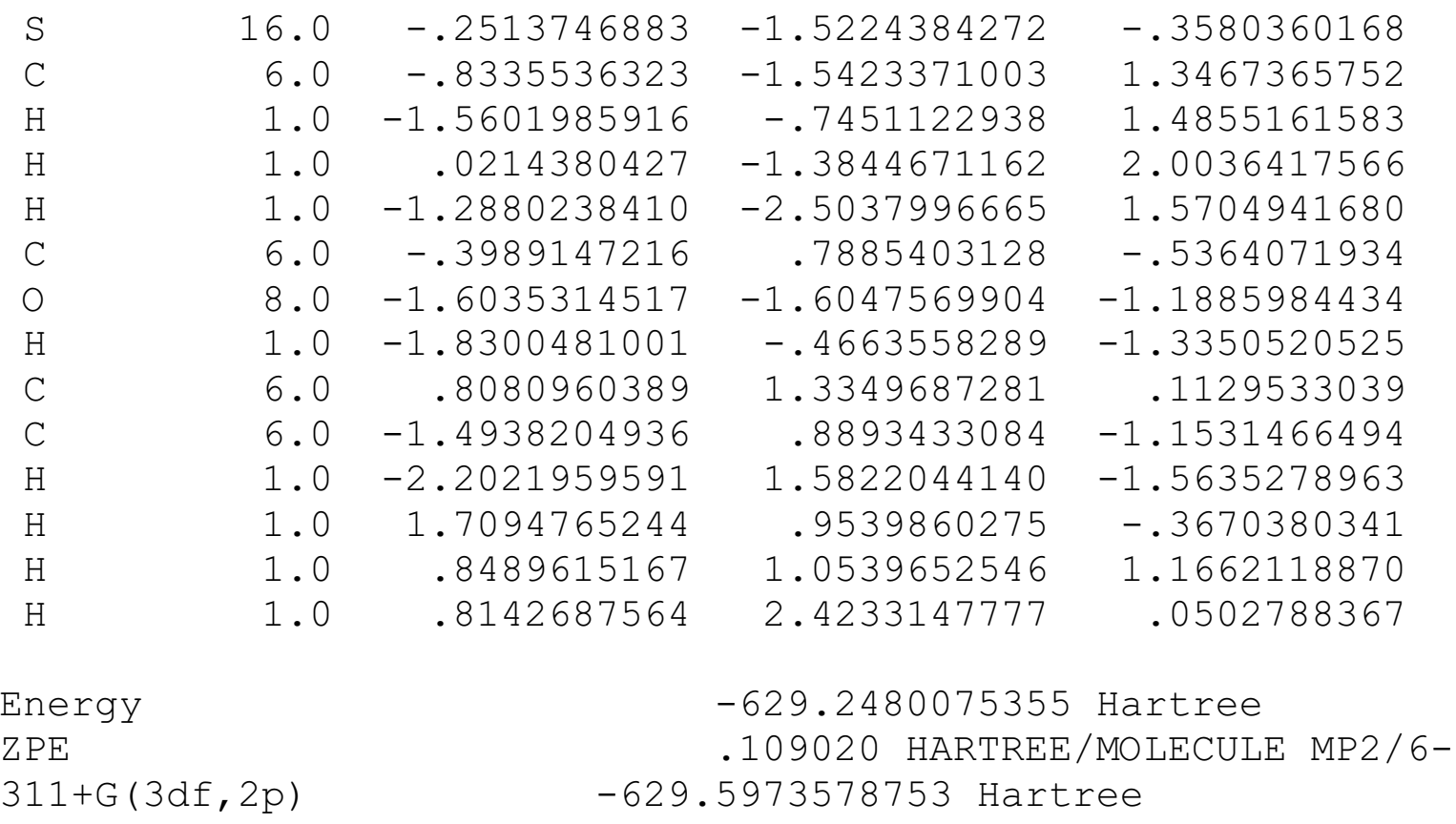




\section{(E)-methanesulfinylpropene TS (H-Transfer)}

$\mathrm{HF} / 6-31 \mathrm{G}(\mathrm{d}, \mathrm{p})$

$\begin{array}{rrrrr}\mathrm{S} & 16.0 & -.4778581302 & -1.4440184371 & -.4157408738 \\ \mathrm{C} & 6.0 & -.9454180319 & -1.4721289852 & 1.3155800140 \\ \mathrm{H} & 1.0 & -1.7600156898 & -.7795584450 & 1.4761366965 \\ \mathrm{H} & 1.0 & -.0824623871 & -1.1689895072 & 1.8979908111 \\ \mathrm{H} & 1.0 & -1.2390960249 & -2.4733846616 & 1.6002454204 \\ \mathrm{C} & 6.0 & -.6150510165 & .6797987956 & -.7829319385 \\ \mathrm{O} & 8.0 & -1.8550105596 & -1.7172208680 & -1.1414991392 \\ \mathrm{H} & 1.0 & -2.1682970659 & -.7626728937 & -1.3793739098 \\ \mathrm{H} & 1.0 & .3177435681 & 1.0394776011 & -.4134502803 \\ \mathrm{C} & 6.0 & -1.7288023093 & .8667243792 & -1.3417986782 \\ \mathrm{C} & 6.0 & -2.4587922889 & 2.0722766498 & -1.8450024531 \\ \mathrm{H} & 1.0 & -1.9202628568 & 3.0062243773 & -1.7061488595 \\ \mathrm{H} & 1.0 & -3.4186778571 & 2.1539546230 & -1.3444803987 \\ \mathrm{H} & 1.0 & -2.6712106500 & 1.9540082719 & -2.9028677108\end{array}$

Energy

$\mathrm{ZPE}$

$\operatorname{MP} 2 / 6-31 G(d, p)$

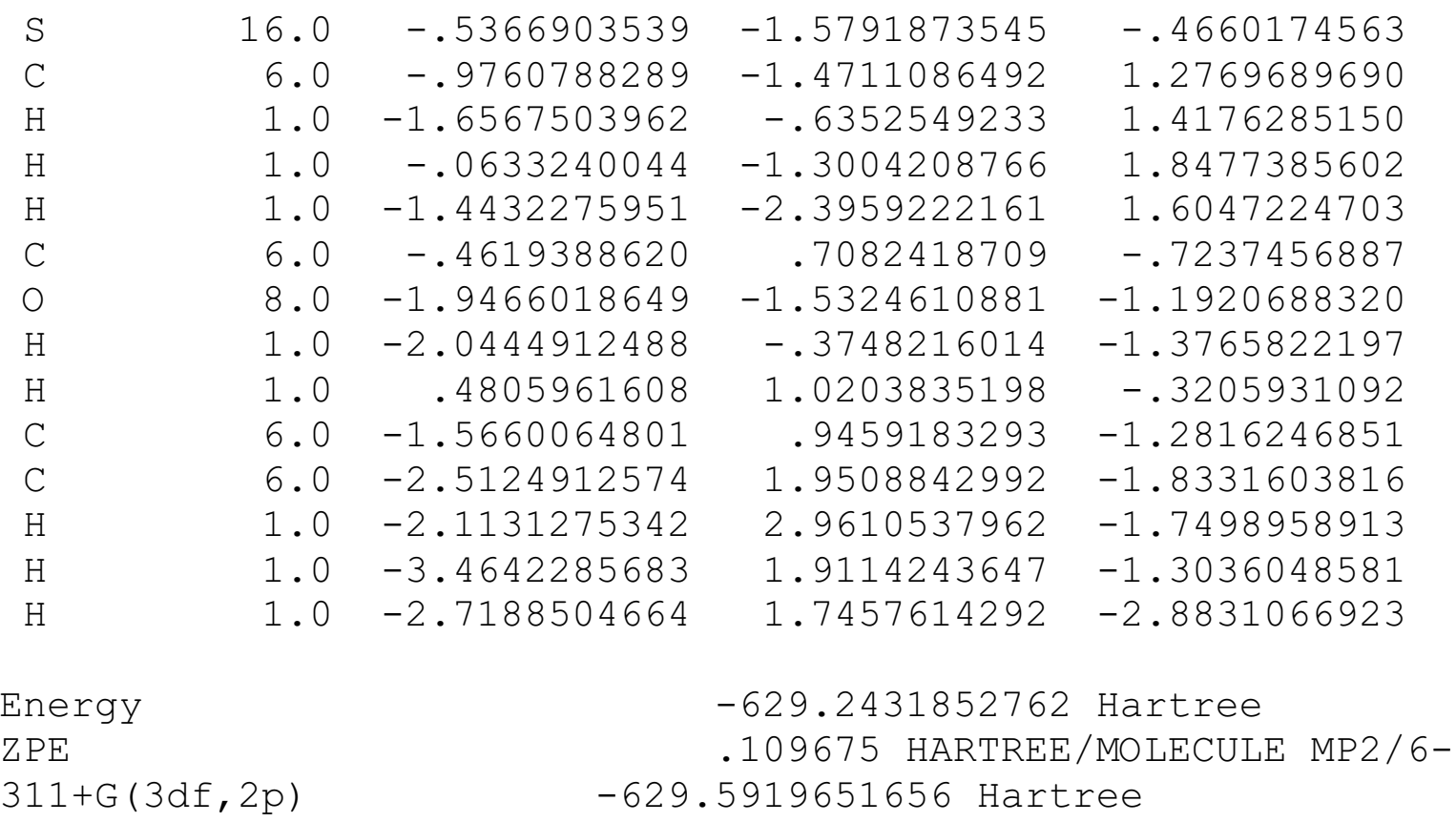

-628.3451644036 Hartree .116122 HARTREE/MOLECULE 


\section{(z) -methanesulfinylpropene}

$\mathrm{HF} / 6-31 \mathrm{G}(\mathrm{d}, \mathrm{p})$

$\begin{array}{lrr}\mathrm{C} & 6.0 & -.4415259919 \\ \mathrm{C} & 6.0 & -.0093587891 \\ \mathrm{H} & 1.0 & -.0009906595 \\ \mathrm{H} & 1.0 & -.2889310353 \\ \mathrm{H} & 1.0 & .8275852313 \\ \mathrm{C} & 6.0 & -1.5753572690 \\ \mathrm{~S} & 16.0 & -2.5571786499 \\ \mathrm{C} & 6.0 & -3.7833304812 \\ \mathrm{H} & 1.0 & -4.4060767903 \\ \mathrm{H} & 1.0 & -3.2719535721 \\ \mathrm{H} & 1.0 & -4.3902316132 \\ \mathrm{O} & 8.0 & -1.6674334879 \\ \mathrm{H} & 1.0 & -2.0434496096 \\ \mathrm{H} & 1.0 & .2023445577\end{array}$

Energy

ZPE

$\operatorname{MP} 2 / 6-31 G(d, p)$

\begin{tabular}{lrrrr} 
C & 6.0 & -.5258436438 & .8842511082 & -.1476436368 \\
$\mathrm{C}$ & 6.0 & -.0115534948 & -.1569698365 & -2.1150075361 \\
$\mathrm{H}$ & 1.0 & .0158302620 & .8683742835 & -2.4717744256 \\
$\mathrm{H}$ & 1.0 & -.0882967482 & -.8398593372 & -2.9526190417 \\
$\mathrm{H}$ & 1.0 & .8534537400 & -.4115897901 & -1.5104459801 \\
$\mathrm{C}$ & 6.0 & -1.5090318394 & .5720953215 & .5505841204 \\
$\mathrm{~S}$ & 16.0 & -2.7448920826 & -.9427015333 & -.7273145683 \\
$\mathrm{C}$ & 6.0 & -3.8224792773 & .4085894322 & -1.2356458496 \\
$\mathrm{H}$ & 1.0 & -4.4421663159 & .0989818852 & -2.0731874460 \\
$\mathrm{H}$ & 1.0 & -3.1976794399 & 1.2512493935 & -1.5215210604 \\
$\mathrm{H}$ & 1.0 & -4.4530265032 & .6906550302 & -.3924346241 \\
$\mathrm{O}$ & 8.0 & -1.7376423609 & -1.0716291046 & -1.9119164438 \\
$\mathrm{H}$ & 1.0 & -2.1065218066 & .6459635553 & 1.4339258353 \\
$\mathrm{H}$ & 1.0 & .3639613506 & 1.4357390841 & -.3460373080 \\
& \multicolumn{5}{c}{-629.1436219431 Hartree } \\
Energy & \multicolumn{5}{c}{-109431 HARTREE /MOLECULE MP2/6- } \\
ZPE & \multicolumn{5}{c}{-629.4950661538 Hartree } \\
311+G(3df, $2 \mathrm{p})$ & \multicolumn{3}{c}{}
\end{tabular}

$.7886427793-.1558910178$

$-.1824798335-2.2972363550$

$.8614454231-2.5182979147$

$-.8063057706-3.1226474138$

$-.5331283194-1.7370765832$

$.4720204517 \quad .4136415436$

$-.6823308427-.5647391713$

$.4274917491-1.2568418078$

$-.1249706624-1.9483311463$

$1.2341041247-1.7646601563$

$.8210943581-.4497923861$

$-1.0318530645-1.7594168729$

$.7110461569 \quad 1.3560793088$

$1.4783729422 \quad .3841720080$
-628.2396232386 Hartree

.115998 HARTREE/MOLECULE 


\section{Methyl methanesulfenate}

$\mathrm{HF} / 6-31 \mathrm{G}(\mathrm{d}, \mathrm{p})$

\begin{tabular}{|c|c|c|c|c|}
\hline 0 & 8.0 & -1.1133328610 & -0.3716051619 & 0.2714427194 \\
\hline $\mathrm{C}$ & 6.0 & -0.8979069966 & 0.5523114614 & 1.3125676756 \\
\hline $\mathrm{H}$ & 1.0 & -1.2933495364 & 0.0981971117 & 2.2116293253 \\
\hline $\mathrm{H}$ & 1.0 & -1.4155403058 & 1.4857977749 & 1.1212909692 \\
\hline $\mathrm{H}$ & 1.0 & 0.1594254911 & 0.7555405361 & 1.4516631115 \\
\hline S & 16.0 & -0.6279307420 & 0.1028110361 & -1.2326126333 \\
\hline $\mathrm{C}$ & 6.0 & 1.0434773512 & -0.5591291869 & -1.2605568864 \\
\hline $\mathrm{H}$ & 1.0 & 1.4380386849 & -0.3523499029 & -2.2493785925 \\
\hline $\mathrm{H}$ & 1.0 & 1.0279228390 & -1.6286993909 & -1.1005703315 \\
\hline $\mathrm{H}$ & 1.0 & 1.6791961276 & -0.0828741706 & -0.5254752523 \\
\hline Energy & & & -551.5661258502 & Hartree \\
\hline $\mathrm{ZPE}$ & & & 0.086089 HARTRE & E/MOLECULE \\
\hline \multicolumn{5}{|c|}{$\operatorname{MP} 2 / 6-31 G(d, p)$} \\
\hline 0 & 8.0 & -1.1656127152 & -0.4091761052 & 0.2649671396 \\
\hline $\mathrm{C}$ & 6.0 & -0.8804074335 & 0.5460266784 & 1.2962526503 \\
\hline $\mathrm{H}$ & 1.0 & -1.2531194431 & 0.1010962114 & 2.2158278297 \\
\hline $\mathrm{H}$ & 1.0 & -1.3880761483 & 1.4922910091 & 1.1100119978 \\
\hline $\mathrm{H}$ & 1.0 & 0.1918258875 & 0.7270161870 & 1.3894748590 \\
\hline $\mathrm{S}$ & 16.0 & -0.6487224158 & 0.1019405148 & -1.2610159122 \\
\hline $\mathrm{C}$ & 6.0 & 1.0272395494 & -0.5444897632 & -1.2378223509 \\
\hline $\mathrm{H}$ & 1.0 & 1.4572521916 & -0.3310232886 & -2.2163787848 \\
\hline $\mathrm{H}$ & 1.0 & 1.0188413489 & -1.6199776635 & -1.0820898721 \\
\hline $\mathrm{H}$ & 1.0 & 1.6407792304 & -0.0637036731 & -0.4792274514 \\
\hline \multirow{2}{*}{\multicolumn{3}{|c|}{$\begin{array}{l}\text { Energy } \\
\text { ZPE }\end{array}$}} & \multicolumn{2}{|c|}{-552.1690358081 Hartree } \\
\hline & \multicolumn{2}{|c|}{$\mathrm{ZPE}$} & \multicolumn{2}{|c|}{$\begin{array}{l}0.082989 \text { HARTREE/MOLECULE MP2/6- } \\
.4288311580 \text { Hartree }\end{array}$} \\
\hline
\end{tabular}




\section{Propyne}

$\mathrm{HF} / 6-31 \mathrm{G}(\mathrm{d}, \mathrm{p})$

$\begin{array}{lrrrr}\mathrm{C} & 6.0 & .0000000001 & -.1033741219 & .2163365067 \\ \mathrm{C} & 6.0 & .0000000000 & -.0243101061 & -.9682368835 \\ \mathrm{C} & 6.0 & .0000000002 & -.2008234710 & 1.6804472048 \\ \mathrm{H} & 1.0 & -.0000000001 & .0459188556 & -2.0223663283 \\ \mathrm{H} & 1.0 & .0000000002 & .7865768377 & 2.1280381582 \\ \mathrm{H} & 1.0 & .8789666549 & -.7324939970 & 2.0268906713 \\ \mathrm{H} & 1.0 & -.8789666551 & -.7324939973 & 2.0268906707\end{array}$

Energy

$\mathrm{ZPE}$

-115.8712460184 Hartree .059777 HARTREE/MOLECULE

MP2 / 6-31G (d, p)

$\begin{array}{lrrrr}\text { C } & 6.0 & .0000000002 & -.1040096863 & .2267895793 \\ \mathrm{C} & 6.0 & .0000000000 & -.0228595529 & -.9904031898 \\ \mathrm{C} & 6.0 & .0000000004 & -.2011842875 & 1.6857842343 \\ \mathrm{H} & 1.0 & -.0000000002 & .0477678256 & -2.0502155819 \\ \mathrm{H} & 1.0 & -.0000000003 & .7886461492 & 2.1396364129 \\ \mathrm{H} & 1.0 & .8814411695 & -.7346802243 & 2.0382042730 \\ \mathrm{H} & 1.0 & -.8814411694 & -.7346802238 & 2.0382042720 \\ & & & \\ \text { Energy } & & -116.2729956353 & \text { Hartree } \\ \text { ZPE } & & .056172 \text { HARTREE/MOLECULE } & \text { MP2/6- } \\ 311+\mathrm{G}(3 \mathrm{df}, 2 \mathrm{p}) & -116.3900121684 & \text { Hartree }\end{array}$




\section{2-methanesulfinylethylsilane}

$\mathrm{HF} / 6-31 \mathrm{G}(\mathrm{d}, \mathrm{p})$

$\begin{array}{lrrrr}\mathrm{S} & 16.0 & -.0025329569 & .1188298676 & -.8128228440 \\ \mathrm{C} & 6.0 & .0249943759 & .0151100303 & .9794635720 \\ \mathrm{H} & 1.0 & .5146707228 & .9108809459 & 1.3381778168 \\ \mathrm{H} & 1.0 & .5885548313 & -.8554371557 & 1.2938342235 \\ \mathrm{H} & 1.0 & -.9830430060 & -.0139082097 & 1.3698783955 \\ \mathrm{SI} & 14.0 & -3.2236732244 & -3.0009892097 & -1.4944128596 \\ \mathrm{O} & 8.0 & -.9546165893 & 1.2083860382 & -1.1594096719 \\ \mathrm{C} & 6.0 & -.8355972403 & -1.4466096627 & -1.1992777113 \\ \mathrm{H} & 1.0 & -.7418217077 & -1.5275744270 & -2.2772360118 \\ \mathrm{C} & 6.0 & -2.3041878699 & -1.5036007782 & -.7795245315 \\ \mathrm{H} & 1.0 & -.2415490645 & -2.2437502668 & -.7624506582 \\ \mathrm{H} & 1.0 & -2.7953592728 & -.5926827824 & -1.1079492889 \\ \mathrm{H} & 1.0 & -2.3953139781 & -1.5381296691 & .3028287576 \\ \mathrm{H} & 1.0 & -3.1747500579 & -2.9713512953 & -2.9745534785 \\ \mathrm{H} & 1.0 & -4.6357830172 & -2.9903933174 & -1.0502558929 \\ \mathrm{H} & 1.0 & -2.5859380450 & -4.2577524081 & -1.0314530169\end{array}$

Energy

$\mathrm{ZPE}$

$\begin{array}{crr}\text { MP2 } / 6-31 G(\mathrm{~d}, \mathrm{p}) & \\ \mathrm{S} & 16.0 & -.0205979048 \\ \mathrm{C} & 6.0 & .0103104783 \\ \mathrm{H} & 1.0 & .4560228237 \\ \mathrm{H} & 1.0 & .6166317719 \\ \mathrm{H} & 1.0 & -1.0032460507 \\ \mathrm{SI} & 14.0 & -3.2186500493 \\ \mathrm{O} & 8.0 & -1.0394450573 \\ \mathrm{C} & 6.0 & -.8193130871 \\ \mathrm{H} & 1.0 & -.7249551345 \\ \mathrm{C} & 6.0 & -2.2799836418 \\ \mathrm{H} & 1.0 & -.2149229725 \\ \mathrm{H} & 1.0 & -2.7470549324 \\ \mathrm{H} & 1.0 & -2.3691509433 \\ \mathrm{H} & 1.0 & -3.1555339072 \\ \mathrm{H} & 1.0 & -4.6328883018 \\ \mathrm{H} & 1.0 & -2.6031691913\end{array}$

-880.6621832552 Hartree

.132001 HARTREE/MOLECULE

$\begin{array}{rr}.1426448816 & -.8403981267 \\ -.0036802961 & .9618735789 \\ .9106436136 & 1.3457688020 \\ -.8593193212 & 1.2551216906 \\ -.0938336551 & 1.3452255124 \\ -2.9745468643 & -1.4860133250 \\ 1.2092554474 & -1.1841425719 \\ -1.4705447594 & -1.1882976278 \\ -1.5791442552 & -2.2705226924 \\ -1.5009127200 & -.7648532027 \\ -2.2505049480 & -.7204811397 \\ -.5702743454 & -1.0982007794 \\ -1.5285164783 & .3225028645 \\ -2.9319338187 & -2.9637972384 \\ -2.9476082333 & -1.0536625020 \\ -4.2406965476 & -1.0252864423\end{array}$

Energy

$\mathrm{ZPE}$

$311+\mathrm{G}(3 \mathrm{df}, 2 \mathrm{p})$

MP2 / G3Large

MP2/G3Large on C also
-881.5152183683 Hartree

.127650 HARTREE/MOLECULE MP2/6-881.8877948984 Hartree

-882.5696425411 Hartree

-882.6272469896 Hartree 


\section{1-methanesulfinylethylsilane}

$\mathrm{HF} / 6-31 \mathrm{G}(\mathrm{d}, \mathrm{p})$

\begin{tabular}{|c|c|c|c|c|}
\hline S & 16.0 & .3366239171 & -.1880774647 & -.6557954123 \\
\hline $\mathrm{C}$ & 6.0 & -.3957474443 & .1035360629 & .9582082805 \\
\hline $\mathrm{H}$ & 1.0 & .2180420122 & .8482343782 & 1.4482868863 \\
\hline $\mathrm{H}$ & 1.0 & -.3921470161 & -.8093092330 & 1.5420108400 \\
\hline $\mathrm{H}$ & 1.0 & -1.3986646223 & .4913796846 & .848153954 \\
\hline $\mathrm{H}$ & 1.0 & -2.7876766421 & -1.9225020433 & -1.808061843 \\
\hline O & 8.0 & .0333107456 & 1.0189613104 & -1.476404452 \\
\hline $\mathrm{C}$ & 6.0 & -.7214394347 & -1.5110687999 & -1.292291889 \\
\hline SI & 14.0 & -.1757334870 & -1.9116497392 & -3.089466127 \\
\hline $\mathrm{C}$ & 6.0 & -2.2213615872 & -1.1857209223 & -1.245973564 \\
\hline $\mathrm{H}$ & 1.0 & -.4961906596 & -2.3870303856 & -.686524639 \\
\hline $\mathrm{H}$ & 1.0 & -2.4043699493 & -.2125628998 & -1.685289702 \\
\hline $\mathrm{H}$ & 1.0 & -2.6110430438 & -1.1899900045 & -.234205235 \\
\hline $\mathrm{H}$ & 1.0 & -.7980709457 & -.9760560397 & -4.042682721 \\
\hline $\mathrm{H}$ & 1.0 & -.6478876402 & -3.2858259830 & -3.381101850 \\
\hline $\mathrm{H}$ & 1.0 & 1.2973614976 & -1.8683559212 & -3.198759022 \\
\hline Y & & & -880 & Har \\
\hline$P E$ & & & .1318 & $\mathrm{MOL}$ \\
\hline
\end{tabular}

$\operatorname{MP} 2 / 6-31 G(d, p)$

$\begin{array}{lrrrr}\mathrm{S} & 16.0 & .3598266764 & -.1893184861 & -.6733986450 \\ \mathrm{C} & 6.0 & -.4154566723 & .0902501324 & .9368572666 \\ \mathrm{H} & 1.0 & .1804090323 & .8429036808 & 1.4480033927 \\ \mathrm{H} & 1.0 & -.4186798165 & -.8325691320 & 1.5150464023 \\ \mathrm{H} & 1.0 & -1.4245682215 & .4689638642 & .7963014629 \\ \mathrm{H} & 1.0 & -2.7862547818 & -1.8682277786 & -1.8282119709 \\ \mathrm{O} & 8.0 & .0369771091 & 1.0194192007 & -1.5324823555 \\ \mathrm{C} & 6.0 & -.7120261650 & -1.5342376743 & -1.2749108033 \\ \mathrm{SI} & 14.0 & -.1834921043 & -1.9140656873 & -3.0734937115 \\ \mathrm{C} & 6.0 & -2.1972644571 & -1.1754243655 & -1.2264889963 \\ \mathrm{H} & 1.0 & -.4832669676 & -2.4096988608 & -.6581919798 \\ \mathrm{H} & 1.0 & -2.3338692195 & -.1742847670 & -1.6356223638 \\ \mathrm{H} & 1.0 & -2.5988223994 & -1.2027040020 & -.2147328319 \\ \mathrm{H} & 1.0 & -.8429642728 & -.9799317552 & -4.0030556571 \\ \mathrm{H} & 1.0 & -.6314287509 & -3.2941123040 & -3.3704896840 \\ \mathrm{H} & 1.0 & 1.2858867111 & -1.8330000654 & -3.2050260255\end{array}$


Energy

$\mathrm{ZPE}$

$311+\mathrm{G}(3 \mathrm{df}, 2 \mathrm{p})$

MP2/G3Large
-881.5148264612 Hartree

.127400 HARTREE/MOLECULE MP2/6-881.8877490318 Hartree

-882.5700384610 Hartree

\section{2-methanesulfinylethylsilane TS (H-Transfer)}

$\begin{array}{lrrrr}\mathrm{HF} / 6-31 \mathrm{G}(\mathrm{d}, \mathrm{p}) & & & \\ \mathrm{S} & 16.0 & -0.9827724727 & -0.6127226969 & 1.2112887883 \\ \mathrm{C} & 6.0 & -0.0906197116 & -2.0252802044 & 1.8678425377 \\ \mathrm{H} & 1.0 & -0.7388588515 & -2.8910340975 & 1.8617847284 \\ \mathrm{H} & 1.0 & 0.2030359354 & -1.7996183428 & 2.8869313308 \\ \mathrm{H} & 1.0 & 0.7845765586 & -2.2242343488 & 1.2627342435 \\ \mathrm{H} & 1.0 & -0.2496196025 & -0.4361839943 & -0.7763339265 \\ \mathrm{O} & 8.0 & -1.1886203028 & -0.9772701500 & -0.2834392211 \\ \mathrm{C} & 6.0 & 0.7272570123 & 0.6699033925 & 0.7209063178 \\ \mathrm{H} & 1.0 & 0.2871667264 & 1.6154725755 & 0.9909307830 \\ \mathrm{C} & 6.0 & 0.9244677507 & 0.3564812800 & -0.6563766667 \\ \mathrm{H} & 1.0 & 1.4244411568 & 0.3151368725 & 1.4629180364 \\ \mathrm{SI} & 14.0 & 0.7041834993 & 1.6780877957 & -1.9502323136 \\ \mathrm{H} & 1.0 & 1.7268020737 & -0.3491379536 & -0.8351484681 \\ \mathrm{H} & 1.0 & -0.4456490158 & 2.5370830523 & -1.5673902543 \\ \mathrm{H} & 1.0 & 0.4345489533 & 1.0778525612 & -3.2785155329 \\ \mathrm{H} & 1.0 & 1.8856215904 & 2.5680537585 & -2.1085197828\end{array}$

Energy

$\mathrm{ZPE}$

$\begin{array}{crr}\text { MP2 / 6-31G }(\mathrm{d}, \mathrm{p}) & \\ \mathrm{S} & 16.0 & -1.0581031798 \\ \mathrm{C} & 6.0 & -0.1048160586 \\ \mathrm{H} & 1.0 & -0.6854921743 \\ \mathrm{H} & 1.0 & 0.1220796547 \\ \mathrm{H} & 1.0 & 0.8191028595 \\ \mathrm{H} & 1.0 & -0.1883461731 \\ \mathrm{O} & 8.0 & -1.1628900250 \\ \mathrm{C} & 6.0 & 0.7657985113 \\ \mathrm{H} & 1.0 & 0.3138870614 \\ \mathrm{C} & 6.0 & 0.9678395491 \\ \mathrm{H} & 1.0 & 1.4289262989 \\ \mathrm{SI} & 14.0 & 0.6813849444 \\ \mathrm{H} & 1.0 & 1.7521037652 \\ \mathrm{H} & 1.0 & -0.4155090522 \\ \mathrm{H} & 1.0 & 0.2925437771 \\ \mathrm{H} & 1.0 & 1.8774515416\end{array}$

-880.5961422286 Hartree

0.126106 HARTREE/MOLECULE

$-0.6190038338$

$-1.9983321327$

$-2.9136047922$

$-1.7919221616$

$-2.1026753502$

$-0.4176044756$

$-0.9684738798$

0.6916603870

1.6448578869

0.3517759044

0.3101432504

1.6476132493

$-0.3801704299$

2.5264371903

1.0328351020

2.4890535855
1.1836983339

1.8384865721

1.7629264664

2.8842080155

1.2731761426

$-0.7861465473$

$-0.3601184651$

0.7358271447

0.9890855086

$-0.6204461333$

1.5060469324

$-1.9317815585$

$-0.7999995394$

$-1.4637764523$

$-3.2214748136$

$-2.1803310068$ 


\section{Energy}

$\mathrm{ZPE}$

$311+G(3 d f, 2 p)$

MP2 / G3Large

MP2/G3Large on $\mathrm{C}$ as well
-881.4717449415 Hartree

0.121545 HARTREE/MOLECULE MP2/6-881.8388713332 Hartree

-882.5207198447 Hartree

-882.5787982013 Hartree

\section{2-methanesulfinylethylsilane TS (Silyl-Transfer)}

$\mathrm{HF} / 6-31 \mathrm{G}(\mathrm{d}, \mathrm{p})$

$\begin{array}{lrrrr}\mathrm{S} & 16.0 & -0.9744792812 & 0.4747020618 & 0.8823187459 \\ \mathrm{C} & 6.0 & -0.2277682712 & -0.5159711908 & 2.1779746869 \\ \mathrm{H} & 1.0 & -0.8431693931 & -1.3864428174 & 2.3591049590 \\ \mathrm{H} & 1.0 & -0.1891893212 & 0.0939437428 & 3.0735315928 \\ \mathrm{H} & 1.0 & 0.7701708120 & -0.8247134128 & 1.8973085489 \\ \mathrm{SI} & 14.0 & 0.3355981354 & -1.0830668075 & -1.3933775626 \\ \mathrm{O} & 8.0 & -1.0039423365 & -0.5089824955 & -0.3419598722 \\ \mathrm{C} & 6.0 & 0.7758585371 & 1.6560296113 & 0.2085580110 \\ \mathrm{H} & 1.0 & 0.2277892584 & 2.5759914776 & 0.1065259328 \\ \mathrm{C} & 6.0 & 1.2390555506 & 0.9848590896 & -0.9401830609 \\ \mathrm{H} & 1.0 & 1.3364958280 & 1.5989456611 & 1.1271794126 \\ \mathrm{H} & 1.0 & 1.0304935822 & 1.4853862705 & -1.8712703760 \\ \mathrm{H} & 1.0 & 2.2405496942 & 0.5901321862 & -0.8730037595 \\ \mathrm{H} & 1.0 & -0.5673971175 & -2.2109844929 & -1.7880174038 \\ \mathrm{H} & 1.0 & 1.3847036778 & -1.7501482990 & -0.5809238845 \\ \mathrm{H} & 1.0 & 0.8358556451 & -0.7317656850 & -2.7531269705 \\ & & & -8.6029451172 & \text { Hartree } \\ \text { Energy } & & & 0.130350 \text { HARTREE/MOLECULE }\end{array}$




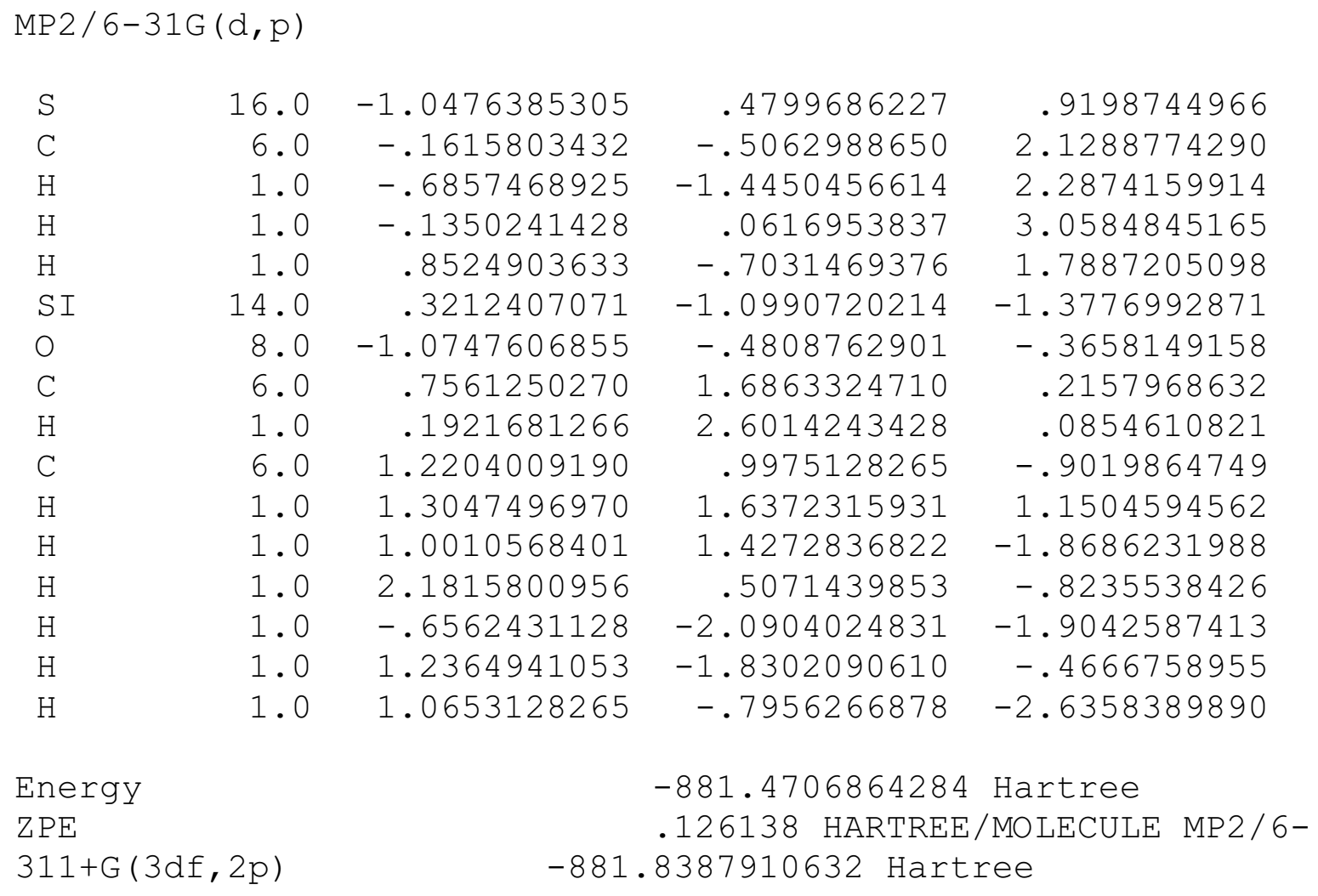

\section{1-methanesulfinylethylsilane TS (H-Transfer)}

$\mathrm{HF} / 6-31 \mathrm{G}(\mathrm{d}, \mathrm{p})$

$\begin{array}{lrrrr}\mathrm{S} & 16.0 & -1.0331167039 & -1.0642982829 & .7778642570 \\ \mathrm{C} & 6.0 & .4057005226 & -1.0917868712 & 1.8547243440 \\ \mathrm{H} & 1.0 & .6956438417 & -2.1173295088 & 2.0409144564 \\ \mathrm{H} & 1.0 & .1400500367 & -.6160335328 & 2.7921961694 \\ \mathrm{H} & 1.0 & 1.2299101432 & -.5648392456 & 1.3916289511 \\ \mathrm{H} & 1.0 & -.2580602545 & -.4724502150 & -1.1531559424 \\ \mathrm{O} & 8.0 & -.4917213441 & -1.5602472971 & -.5824490067 \\ \mathrm{C} & 6.0 & -.8272964912 & 1.1488538869 & .0245761725 \\ \mathrm{SI} & 14.0 & -2.6140082894 & 1.7367491785 & .1972615781 \\ \mathrm{C} & 6.0 & -.2410473983 & .8735753691 & -1.2352450036 \\ \mathrm{H} & 1.0 & -.1633891531 & 1.4483197180 & .8215919827 \\ \mathrm{H} & 1.0 & -.8364343967 & 1.0788038706 & -2.1142569730 \\ \mathrm{H} & 1.0 & .8050239140 & 1.1180142514 & -1.3519101516 \\ \mathrm{H} & 1.0 & -2.6908596371 & 3.1750826244 & -.1492431477 \\ \mathrm{H} & 1.0 & -3.0678762604 & 1.5567395012 & 1.5941066007 \\ \mathrm{H} & 1.0 & -3.4749544785 & .9632237373 & -.7201142708 \\ & & & -8.5875338195 & \text { Hartree } \\ \text { Energy } & & & .125448 \text { HARTREE/MOLECULE }\end{array}$




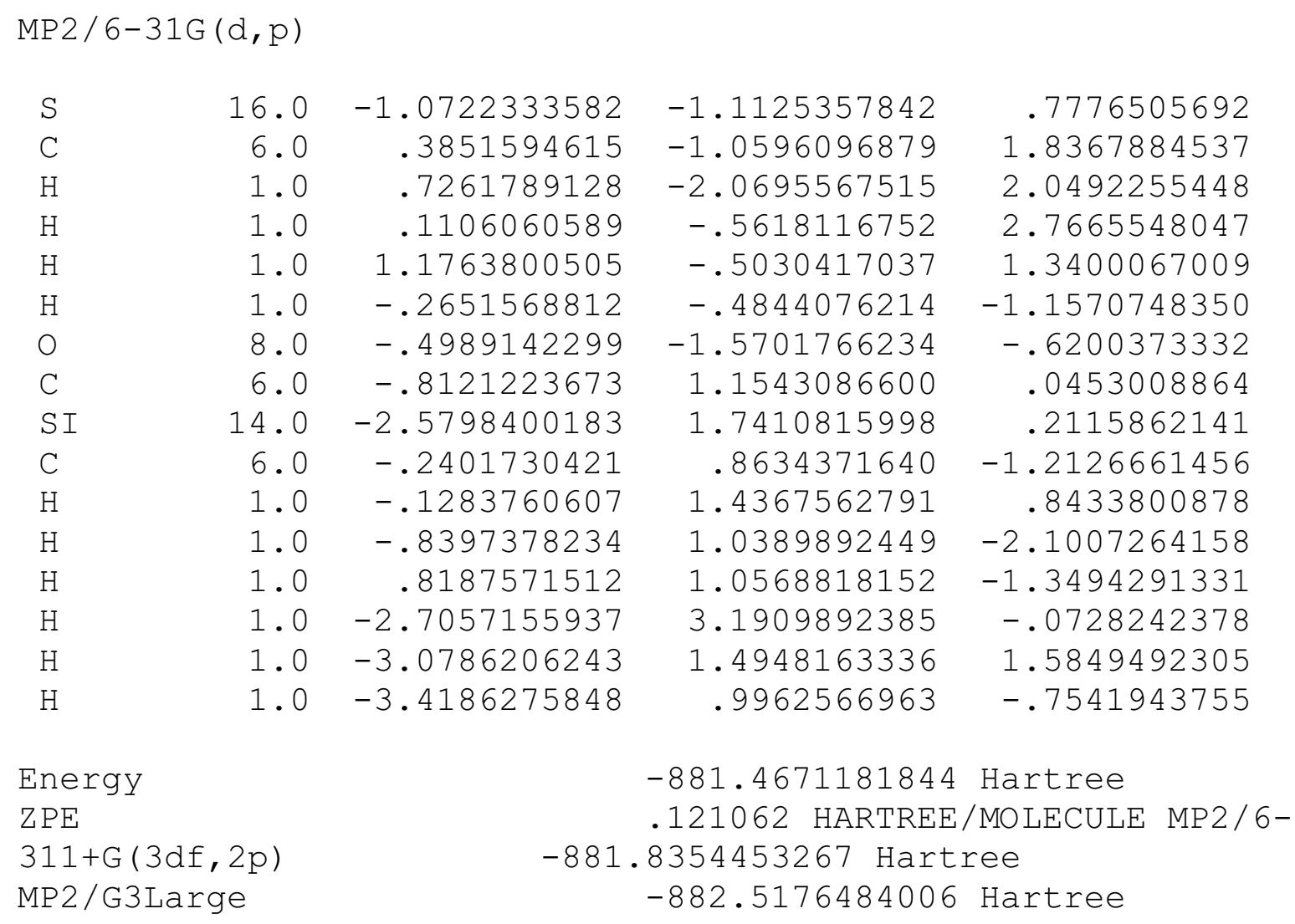

\section{Vinyl silane}

$\mathrm{HF} / 6-31 \mathrm{G}(\mathrm{d}, \mathrm{p})$

$\begin{array}{lrrrr}\text { C } & 6.0 & -.9617102281 & -.3187948283 & .4770779461 \\ \mathrm{C} & 6.0 & -.2249855759 & .2128585582 & -.4862789449 \\ \mathrm{SI} & 14.0 & 1.6391700726 & .3958006377 & -.3828688504 \\ \mathrm{H} & 1.0 & -2.0326681141 & -.4118259760 & .3957126523 \\ \mathrm{H} & 1.0 & -.5282112762 & -.6863872877 & 1.3926169619 \\ \mathrm{H} & 1.0 & -.7310390773 & .5590923283 & -1.3763311007 \\ \mathrm{H} & 1.0 & 2.0351318485 & 1.8200470767 & -.4898933585 \\ \mathrm{H} & 1.0 & 2.3036559643 & -.3411948617 & -1.4838317006 \\ \mathrm{H} & 1.0 & 2.1076563862 & -.1425956472 & .9147963948 \\ & & & -368.1208481819 & \text { Hartree } \\ \text { Energy } & & & .071451 \text { HARTREE/MOLECULE } \\ \text { ZPE } & & & \end{array}$




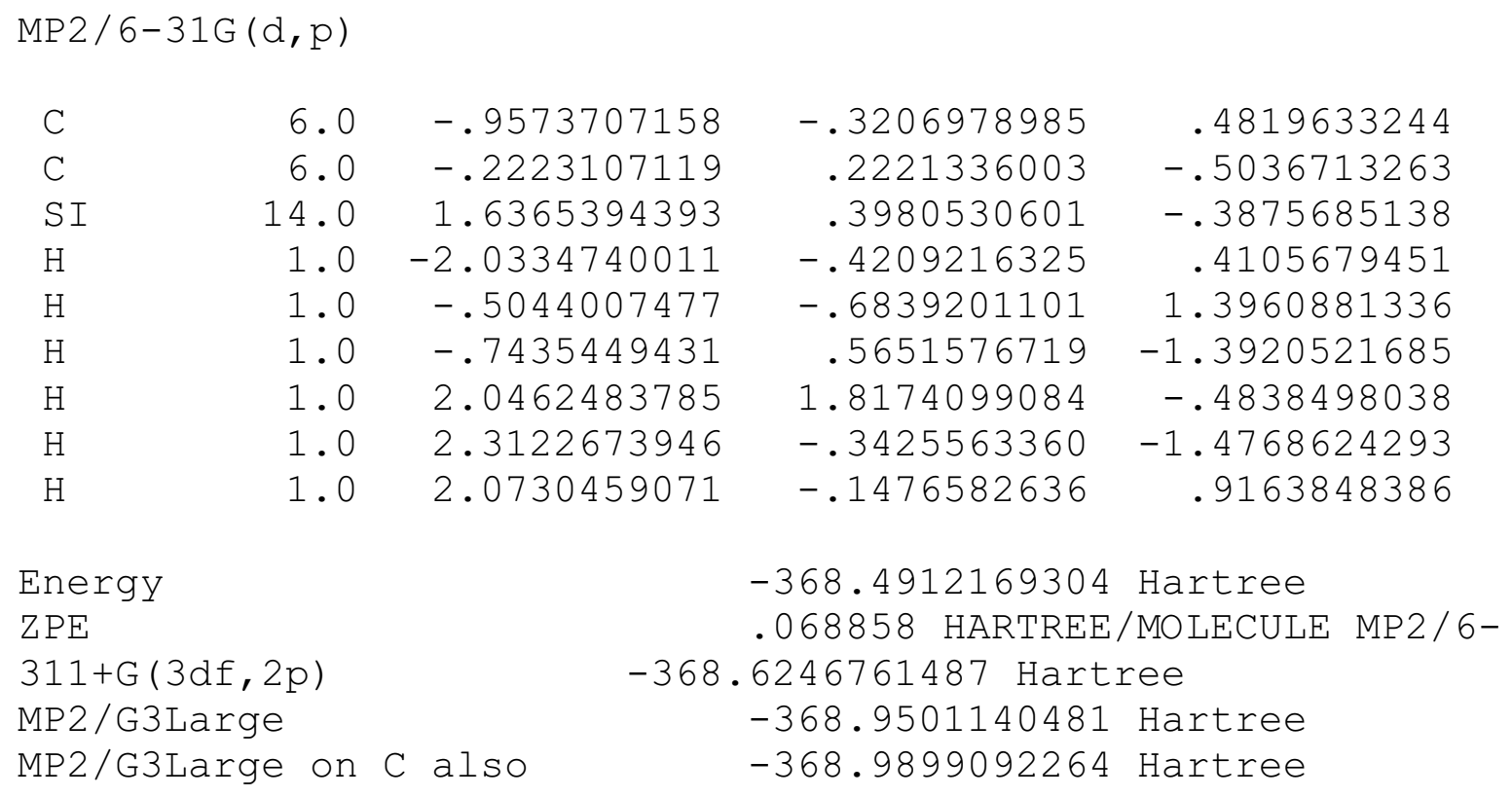

\section{Methanesulfenic acid}

$\mathrm{HF} / 6-31 \mathrm{G}(\mathrm{d}, \mathrm{p})$

\begin{tabular}{|c|c|c|c|c|}
\hline S & 16.0 & .0800853973 & .0189420622 & -.7668909104 \\
\hline $\mathrm{C}$ & 6.0 & .0323639744 & -.0528983013 & 1.0275143005 \\
\hline $\mathrm{H}$ & 1.0 & -.3691487336 & .8965808781 & 1.3646454971 \\
\hline $\mathrm{H}$ & 1.0 & 1.0295886930 & -.1786570436 & 1.4269357743 \\
\hline $\mathrm{H}$ & 1.0 & -.6105138308 & -.8505755985 & 1.3776682904 \\
\hline O & 8.0 & .8015338285 & -1.4328766444 & -1.1019985369 \\
\hline $\mathrm{H}$ & 1.0 & .1322298712 & -2.0857678525 & -1.2396456150 \\
\hline $\begin{array}{l}\text { Energy } \\
\text { ZPE }\end{array}$ & & & $\begin{array}{l}-512.5418924638 \\
.056226 \text { HARTREE }\end{array}$ & $\begin{array}{l}\text { Hartree } \\
\text { MOLECULE }\end{array}$ \\
\hline \multicolumn{5}{|c|}{$\mathrm{MP} 2 / 6-31 \mathrm{G}(\mathrm{d}, \mathrm{p})$} \\
\hline S & 16.0 & .0697252069 & .0335180435 & -.7690048086 \\
\hline $\mathrm{C}$ & 6.0 & .0297830941 & -.0526385443 & 1.0232589234 \\
\hline $\mathrm{H}$ & 1.0 & -.3698312274 & .8991530378 & 1.3734936641 \\
\hline $\mathrm{H}$ & 1.0 & 1.0332297674 & -.1812418896 & 1.420237766 \\
\hline $\mathrm{H}$ & 1.0 & -.6152925101 & -.8535805781 & 1.377108313 \\
\hline O & 8.0 & .8297324583 & -1.4429941494 & -1.1045458235 \\
\hline 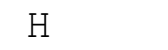 & 1.0 & .1187924108 & -2.0874684200 & -1.232319234 \\
\hline
\end{tabular}


Energy

$\mathrm{ZPE}$

$311+\mathrm{G}(3 \mathrm{df}, 2 \mathrm{p})$

MP2 / G3Large

MP2/G3Large on C also
-513.0011201229 Hartree

.053758 HARTREE/MOLECULE MP2/6-513.2241253557 Hartree

-513.5786479620 Hartree

-513.5978861241 Hartree

\section{Methyl Propyl Sulfoxide}

$\mathrm{HF} / 6-31 \mathrm{G}(\mathrm{d}, \mathrm{p})$

$\begin{array}{lrrrr}\mathrm{S} & 16.0 & .0044037964 & .1002693135 & -.8110406959 \\ \mathrm{C} & 6.0 & .0115179366 & .0033289489 & .9827293705 \\ \mathrm{H} & 1.0 & .5258834362 & .8850068302 & 1.3419121070 \\ \mathrm{H} & 1.0 & .5447786548 & -.8827052436 & 1.3061785752 \\ \mathrm{H} & 1.0 & -1.0001007094 & .0068103924 & 1.3646169420 \\ \mathrm{H} & 1.0 & -2.5709931678 & -3.6399466621 & -1.0569732950 \\ \mathrm{O} & 8.0 & -.9165359376 & 1.2129211358 & -1.1707171524 \\ \mathrm{C} & 6.0 & -.8520505328 & -1.4448930397 & -1.2028064318 \\ \mathrm{H} & 1.0 & -.7750225313 & -1.5232527189 & -2.2825565017 \\ \mathrm{C} & 6.0 & -2.3189322735 & -1.4967425613 & -.7809077864 \\ \mathrm{H} & 1.0 & -.2679063914 & -2.2560193507 & -.7767327433 \\ \mathrm{H} & 1.0 & -2.8001567235 & -.5807176205 & -1.1022135600 \\ \mathrm{H} & 1.0 & -2.3948514312 & -1.5340620982 & .3015793272 \\ \mathrm{C} & 6.0 & -3.0336894525 & -2.7100122865 & -1.3758742265 \\ \mathrm{H} & 1.0 & -3.0142882002 & -2.6833132188 & -2.4613371956 \\ \mathrm{H} & 1.0 & -4.0729494727 & -2.7339297207 & -1.0647298336 \\ & & & -629.6232106417 & \text { Hartree } \\ \text { Energy } & & & .146366 \text { HARTREE/MOLECULE }\end{array}$




$\begin{array}{lrrrr}\text { MP2/6-31G }(\mathrm{d}, \mathrm{p}) & & & \\ \mathrm{S} & 16.0 & -.0033837350 & .1207957275 & -.8393159414 \\ \mathrm{C} & 6.0 & -.0112462256 & -.0101597351 & .9654029119 \\ \mathrm{H} & 1.0 & .4774338481 & .8822924134 & 1.3482488343 \\ \mathrm{H} & 1.0 & .5423324004 & -.8944139682 & 1.2775535149 \\ \mathrm{H} & 1.0 & -1.0343117836 & -.0405646346 & 1.3319908876 \\ \mathrm{H} & 1.0 & -2.5838126933 & -3.6310511572 & -1.0707204322 \\ \mathrm{O} & 8.0 & -.9766557010 & 1.2196483960 & -1.2131687415 \\ \mathrm{C} & 6.0 & -.8309493531 & -1.4667831867 & -1.1903027547 \\ \mathrm{H} & 1.0 & -.7633753909 & -1.5698382959 & -2.2752419916 \\ \mathrm{C} & 6.0 & -2.2887003401 & -1.5016809656 & -.7590814407 \\ \mathrm{H} & 1.0 & -.2327719756 & -2.2626651627 & -.7404646589 \\ \mathrm{H} & 1.0 & -2.7486623406 & -.5609758586 & -1.0653749060 \\ \mathrm{H} & 1.0 & -2.3579094469 & -1.5534588160 & .3283765022 \\ \mathrm{C} & 6.0 & -3.0322084515 & -2.6838872984 & -1.3710477681 \\ \mathrm{H} & 1.0 & -3.0118132251 & -2.6334985265 & -2.4594224548 \\ \mathrm{H} & 1.0 & -4.0748585860 & -2.6910168316 & -1.0563046613 \\ & & & -630.5269986050 & \text { Hartree } \\ \text { Energy } & & & .141710 \text { HARTREE/MOLECULE } \\ \text { ZPE } & & & -630.8833146812 & \text { Hartree } \\ \text { MP2/6-311+G(3df, 2p) } & \end{array}$

\section{Methyl Isopropyl Sulfoxide}

$\mathrm{HF} / 6-31 \mathrm{G}(\mathrm{d}, \mathrm{p})$

$\begin{array}{lrrrr}\mathrm{S} & 16.0 & .2609778701 & -.0729109300 & -.8516989871 \\ \mathrm{C} & 6.0 & -.2757619106 & .0242556702 & .8591483946 \\ \mathrm{H} & 1.0 & .2832824247 & .8301127320 & 1.3164978671 \\ \mathrm{H} & 1.0 & -.0558181999 & -.9039815599 & 1.3732820384 \\ \mathrm{H} & 1.0 & -1.3305776187 & .2557825047 & .9105479176 \\ \mathrm{H} & 1.0 & -2.7386136183 & -2.0648385333 & -1.8596312849 \\ \mathrm{O} & 8.0 & -.2966721452 & 1.1249603545 & -1.5399111818 \\ \mathrm{C} & 6.0 & -.7088553737 & -1.5083707142 & -1.4189849628 \\ \mathrm{C} & 6.0 & -.2384951520 & -1.8339842153 & -2.8388236331 \\ \mathrm{C} & 6.0 & -2.2133502474 & -1.2504559873 & -1.3709790138 \\ \mathrm{H} & 1.0 & -.4343921504 & -2.3228476368 & -.7530139782 \\ \mathrm{H} & 1.0 & -2.4480506413 & -.3278723264 & -1.8873704056 \\ \mathrm{H} & 1.0 & -2.5881424205 & -1.1834490429 & -.3564177333 \\ \mathrm{H} & 1.0 & -.4469373742 & -1.0046083957 & -3.5053448196 \\ \mathrm{H} & 1.0 & -.7602997749 & -2.7103038079 & -3.2082768258 \\ \mathrm{H} & 1.0 & .8267120323 & -2.0375261118 & -2.8689198916 \\ & & & -629.6239780075 & \text { Hartree } \\ \text { Energy } & & & .145884 & \text { HARTREE/MOLECULE } \\ \text { ZPE } & & & \end{array}$




$\begin{array}{lrrrr}\text { MP2/6-31G }(\mathrm{d}, \mathrm{p}) & & \\ \mathrm{S} & 16.0 & .2880957119 & -.0731635949 & -.8712886271 \\ \mathrm{C} & 6.0 & -.3096018466 & .0222390657 & .8332396003 \\ \mathrm{H} & 1.0 & .2444650578 & .8211835069 & 1.3199408051 \\ \mathrm{H} & 1.0 & -.1241165537 & -.9195034424 & 1.3477845765 \\ \mathrm{H} & 1.0 & -1.3681772268 & .2693705394 & .8380121661 \\ \mathrm{H} & 1.0 & -2.7326477234 & -2.0118566114 & -1.8837261319 \\ \mathrm{O} & 8.0 & -.2792577176 & 1.1215009551 & -1.6136921292 \\ \mathrm{C} & 6.0 & -.6900676432 & -1.5322693857 & -1.4054246401 \\ \mathrm{C} & 6.0 & -.2472003921 & -1.8353859927 & -2.8323363264 \\ \mathrm{C} & 6.0 & -2.1827033548 & -1.2508242386 & -1.3313484595 \\ \mathrm{H} & 1.0 & -.3970606463 & -2.3472266351 & -.7372125291 \\ \mathrm{H} & 1.0 & -2.3823230767 & -.2795272864 & -1.7843952155 \\ \mathrm{H} & 1.0 & -2.5554755400 & -1.2460631261 & -.3091798112 \\ \mathrm{H} & 1.0 & -.4774608558 & -.9856737165 & -3.4740447977 \\ \mathrm{H} & 1.0 & -.7752238841 & -2.7101092502 & -3.2091780670 \\ \mathrm{H} & 1.0 & .8237613913 & -2.0287287871 & -2.8870469133 \\ & & & -630.5309128226 & \text { Hartree } \\ \text { Energy } & & & .140909 \text { HARTREE/MOLECULE } \\ \text { ZPE } & & & -630.8876957332 & \text { Hartree } \\ \text { MP2/6-311+G(3df,2p) } & & -631.3042371072 & \text { Hartree } \\ \text { MP2/G3Large } & & \end{array}$

\section{Methyl Propyl Sulfoxide TS (H-transfer)}

$\mathrm{HF} / 6-31 \mathrm{G}(\mathrm{d}, \mathrm{p})$

$\begin{array}{rrrrr}\mathrm{S} & 16.0 & -.9991661606 & -.5472691559 & 1.1148758256 \\ \mathrm{C} & 6.0 & -.1516975325 & -1.9417230471 & 1.8687987506 \\ \mathrm{H} & 1.0 & -.8023576870 & -2.8058603446 & 1.8627141451 \\ \mathrm{H} & 1.0 & .0929093652 & -1.6812516359 & 2.8928230816 \\ \mathrm{H} & 1.0 & .7528926328 & -2.1711908462 & 1.3199012179 \\ \mathrm{H} & 1.0 & -.1215857278 & -.3884041348 & -.8383460513 \\ \mathrm{O} & 8.0 & -1.1225532007 & -.9557212110 & -.3746942895 \\ \mathrm{C} & 6.0 & .8592905581 & .7272860710 & .5831702578 \\ \mathrm{H} & 1.0 & .4694576278 & 1.6698812915 & .9253718062 \\ \mathrm{C} & 6.0 & .9457032513 & .4526370920 & -.7956723722 \\ \mathrm{H} & 1.0 & 1.5309578528 & .2544225862 & 1.2781274046 \\ \mathrm{C} & 6.0 & .6148782679 & 1.5564064213 & -1.7918820356 \\ \mathrm{H} & 1.0 & 1.7727588668 & -.1860130835 & -1.0769524510 \\ \mathrm{H} & 1.0 & -.2951798045 & 2.0768607100 & -1.5058382040 \\ \mathrm{H} & 1.0 & .4512236793 & 1.1459770194 & -2.7827440324 \\ \mathrm{H} & 1.0 & 1.4084293113 & 2.2965517677 & -1.8702724534\end{array}$


Energy

$\mathrm{ZPE}$

MP2 / 6-31G $(\mathrm{d}, \mathrm{p})$
-629.5457112911 Hartree .139644 HARTREE/MOLECULE

$\begin{array}{rrrrr}\mathrm{S} & 16.0 & -1.0553939206 & -0.5377369385 & 1.1005008275 \\ \mathrm{C} & 6.0 & -0.1429269182 & -1.9073451268 & 1.8345165933 \\ \mathrm{H} & 1.0 & -0.7484158694 & -2.8094691383 & 1.8113158375 \\ \mathrm{H} & 1.0 & 0.0902345005 & -1.6487104912 & 2.8672281775 \\ \mathrm{H} & 1.0 & 0.7784954759 & -2.0718324008 & 1.2800301801 \\ \mathrm{H} & 1.0 & -0.1140691912 & -0.3835660359 & -0.8515955811 \\ \mathrm{O} & 8.0 & -1.1339285495 & -0.9351911503 & -0.4278251865 \\ \mathrm{C} & 6.0 & 0.8638089417 & 0.7234950347 & 0.6103262174 \\ \mathrm{H} & 1.0 & 0.4861908252 & 1.6864475311 & 0.9353508917 \\ \mathrm{C} & 6.0 & 0.9535506713 & 0.4406693589 & -0.7639743986 \\ \mathrm{H} & 1.0 & 1.5337921225 & 0.2441730078 & 1.3149251817 \\ \mathrm{C} & 6.0 & 0.6076584531 & 1.5223778844 & -1.7684437863 \\ \mathrm{H} & 1.0 & 1.7551213581 & -0.2331049594 & -1.0562850185 \\ \mathrm{H} & 1.0 & -0.2830745804 & 2.0628426389 & -1.4472225364 \\ \mathrm{H} & 1.0 & 0.3985728383 & 1.1031695061 & -2.7518581556 \\ \mathrm{H} & 1.0 & 1.4163451432 & 2.2463707791 & -1.8776086436\end{array}$

Energy

$\mathrm{ZPE}$

MP2 / 6-311+G (3df, 2p)
-630.4757255639 Hartree

0.134856 HARTREE/MOLECULE -630.8268232990 Hartree

\section{Methyl Propyl Sulfoxide TS (Methyl-transfer)}

$\mathrm{HF} / 6-31 \mathrm{G}(\mathrm{d}, \mathrm{p})$

$\begin{array}{rrrrr}\mathrm{S} & 16.0 & -1.0821839316 & .2478728476 & .9220963824 \\ \mathrm{C} & 6.0 & -.1518416273 & -.4773790091 & 2.2781110264 \\ \mathrm{H} & 1.0 & -.6252785510 & -1.3976731012 & 2.5954435749 \\ \mathrm{H} & 1.0 & -.1330816680 & .2239980376 & 3.1053600590 \\ \mathrm{H} & 1.0 & .8611910326 & -.6912020578 & 1.9569392367 \\ \mathrm{C} & 6.0 & .4627670277 & -1.1521848755 & -1.6124210476 \\ \mathrm{O} & 8.0 & -.9970067871 & -.8029631886 & -.1761993413 \\ \mathrm{C} & 6.0 & .7095843257 & 1.6742279351 & .1077623830 \\ \mathrm{H} & 1.0 & .0234723086 & 2.4664211862 & -.1252768671 \\ \mathrm{C} & 6.0 & 1.3037731567 & .9605222152 & -.8938111737 \\ \mathrm{H} & 1.0 & 1.1575992588 & 1.7271027502 & 1.0835356229 \\ \mathrm{H} & 1.0 & 1.1075883052 & 1.2352961797 & -1.9127940320 \\ \mathrm{H} & 1.0 & 2.2296688142 & .4524246814 & -.6977588945 \\ \mathrm{H} & 1.0 & -.4203278775 & -1.1186627597 & -2.2138078626 \\ \mathrm{H} & 1.0 & .5660368237 & -2.0163185661 & -.9921265606 \\ \mathrm{H} & 1.0 & 1.3586643892 & -.8835673749 & -2.1344135061\end{array}$


Energy

$\mathrm{ZPE}$

$\operatorname{MP} 2 / 6-31 G(d, p)$

$\begin{array}{rrr}\mathrm{S} & 16.0 & -1.1232660766 \\ \mathrm{C} & 6.0 & -.1970942468 \\ \mathrm{H} & 1.0 & -.7034184332 \\ \mathrm{H} & 1.0 & -.1222491572 \\ \mathrm{H} & 1.0 & .7985077109 \\ \mathrm{C} & 6.0 & .4379157823 \\ \mathrm{O} & 8.0 & -1.1222096926 \\ \mathrm{C} & 6.0 & .7875241489 \\ \mathrm{H} & 1.0 & .1585610479 \\ \mathrm{C} & 6.0 & 1.3043061548 \\ \mathrm{H} & 1.0 & 1.2549732026 \\ \mathrm{H} & 1.0 & 1.0563092013 \\ \mathrm{H} & 1.0 & 2.1739834685 \\ \mathrm{H} & 1.0 & -.4015257188 \\ \mathrm{H} & 1.0 & . .9934860161 \\ \mathrm{H} & 1.0 & 1.0748215917\end{array}$

Energy

$\mathrm{ZPE}$

MP2 / 6-311+G (3df, 2p)
-629.4368784316 Hartree .140590 HARTREE/MOLECULE

\section{Methyl Isopropyl Sulfoxide TS (H-transfer)}

$\mathrm{HF} / 6-31 \mathrm{G}(\mathrm{d}, \mathrm{p})$

$\begin{array}{lrrrr}\mathrm{S} & 16.0 & -1.1002214265 & -1.0234046460 & .8201183936 \\ \mathrm{C} & 6.0 & .3891721071 & -1.0569047139 & 1.8248007160 \\ \mathrm{H} & 1.0 & .7120644326 & -2.0807574109 & 1.9580624076 \\ \mathrm{H} & 1.0 & .1609553381 & -.6221644641 & 2.7919317185 \\ \mathrm{H} & 1.0 & 1.1793538338 & -.4934201476 & 1.3442993739 \\ \mathrm{H} & 1.0 & -.3947702157 & -.4354564827 & -1.1250480878 \\ \mathrm{O} & 8.0 & -.6169259516 & -1.5225939267 & -.5677964171 \\ \mathrm{C} & 6.0 & -.9784215621 & 1.1795368807 & .0685554682 \\ \mathrm{C} & 6.0 & -2.4135880469 & 1.6273231192 & .1691607559 \\ \mathrm{C} & 6.0 & -.3787245108 & .9219219838 & -1.1850221478 \\ \mathrm{H} & 1.0 & -.3464023474 & 1.5098523561 & .8762940660 \\ \mathrm{H} & 1.0 & -.9779629243 & 1.1516707645 & -2.0545548413 \\ \mathrm{H} & 1.0 & .6635328555 & 1.1860590515 & -1.2847814638 \\ \mathrm{H} & 1.0 & -2.4632638705 & 2.6863462911 & -.0736670175 \\ \mathrm{H} & 1.0 & -2.8186797277 & 1.4894875326 & 1.1644411939 \\ \mathrm{H} & 1.0 & -3.0385539325 & 1.0948809965 & -.5383041025\end{array}$

.3464568431

$-.4972385748$

$-1.4189105647$

.1593473967

$-.7259925587$

$-1.1554006778$

$-.6874802194$

1.7137082802

2.5751905519

1.0134692648

1.6598886760

1.3075811009 .3899116400

$-1.6890950020$

$-1.7714523532$

$-.7720689028$
.8577307248

2.1509984189

2.4252945934

3. 0171798985

1.7758083362

$-1.4960837304$

$-.3032896998$

.1869095124

.0122850681

$-.8711812562$

1.1621230494

$-1.8777076737$

$-.7430590985$

$-1.9242920267$

$-.7952729707$

$-2.2868041460$

-630.3803027558 Hartree .135119 HARTREE/MOLECULE -630.7341076509 Hartree 
Energy

$\mathrm{ZPE}$

$\operatorname{MP} 2 / 6-31 G(d, p)$

$\begin{array}{lrr}\mathrm{S} & 16.0 & -1.1416565064 \\ \mathrm{C} & 6.0 & .3691196771 \\ \mathrm{H} & 1.0 & .7518401982 \\ \mathrm{H} & 1.0 & .1336103638 \\ \mathrm{H} & 1.0 & 1.1169021193 \\ \mathrm{H} & 1.0 & -.4051312269 \\ \mathrm{O} & 8.0 & -.6377784586 \\ \mathrm{C} & 6.0 & -.9622606308 \\ \mathrm{C} & 6.0 & -2.3932587960 \\ \mathrm{C} & 6.0 & -.3680434183 \\ \mathrm{H} & 1.0 & -.3213502662 \\ \mathrm{H} & 1.0 & -.9655270923 \\ \mathrm{H} & 1.0 & .6898248936 \\ \mathrm{H} & 1.0 & -2.4607648690 \\ \mathrm{H} & 1.0 & -2.8199292314 \\ \mathrm{H} & 1.0 & -3.0080327051\end{array}$

Energy

$\mathrm{ZPE}$

MP 2/6-311+G (3df, 2p)

MP2 / G3Large

\section{Propene}

$\mathrm{HF} / 6-31 \mathrm{G}(\mathrm{d}, \mathrm{p})$

$\begin{array}{lrrrr}\mathrm{C} & 6.0 & -.7777599990 & -.3641775744 & .4278484597 \\ \mathrm{C} & 6.0 & -.0145791520 & .2806161684 & -.4319328997 \\ \mathrm{C} & 6.0 & 1.4807842166 & .3936636759 & -.3492864691 \\ \mathrm{H} & 1.0 & -1.8463119357 & -.4070531562 & .3102409331 \\ \mathrm{H} & 1.0 & -.3644369507 & -.8746728046 & 1.2819106220 \\ \mathrm{H} & 1.0 & -.4789960100 & .7750879872 & -1.2719505269 \\ \mathrm{H} & 1.0 & 1.7877814556 & 1.4348625636 & -.2888399498 \\ \mathrm{H} & 1.0 & 1.9498739470 & -.0252763132 & -1.2360251634 \\ \mathrm{H} & 1.0 & 1.8706444283 & -.1260505467 & .5190349941\end{array}$

Energy

$\mathrm{ZPE}$
-629.5517780930 Hartree .139344 HARTREE/MOLECULE

$-1.0511066112$

.8217153184

$-1.0226363331$

1.8015535818

$-2.0326649306$

1.9210486041

$-.6023104430$

$-.4056779174$

$-.4573622716$

$-1.5284708307$

1.1714701044

1.6280860765

.9191765573

1.4969451424

1.1170180306

1.1252363584

2. 6973347035

2.7791770723

1.3073164070

$-1.1250533550$

$-.6043600357$

.0895854157

.1796560936

$-1.1578622969$

.9047645834

$-2.0413814448$

$-1.2689882713$

$-.0315712090$

1.4514031574

1.1664457826

1.1059363910

$-.5535562302$
-630.4796892378 Hartree .134584 HARTREE/MOLECULE -630.8316072064 Hartree -631.2480691552 Hartree

-117.0816139306 Hartree .084965 HARTREE/MOLECULE 


$\begin{array}{lrrrr}\text { MP2/6-31G }(\mathrm{d}, \mathrm{p}) & & \\ \mathrm{C} & 6.0 & -.7796021629 & -.3674594385 & .4323185757 \\ \mathrm{C} & 6.0 & -.0120519314 & .2887603128 & -.4442776531 \\ \mathrm{C} & 6.0 & 1.4790658250 & .3949332719 & -.3517650496 \\ \mathrm{H} & 1.0 & -1.8532030458 & -.4146209381 & .3204525970 \\ \mathrm{H} & 1.0 & -.3504404901 & -.8751784885 & 1.2861413033 \\ \mathrm{H} & 1.0 & -.4835926003 & . .7843895294 & -1.2866020160 \\ \mathrm{H} & 1.0 & 1.7949693391 & 1.4368293226 & -.2844740760 \\ \mathrm{H} & 1.0 & 1.9583724676 & -.0286016564 & -1.2354092700 \\ \mathrm{H} & 1.0 & 1.8534825989 & -.1320519152 & .5246155887 \\ & & & & \\ \text { Energy } & & & -117.5039138387 & \text { Hartree } \\ \text { ZPE } & & & -117.6224988562 & \text { Hartree } \\ \text { MP2/6-311+G(3df,2p) } & & -117.6827153751 & \text { Hartree } \\ \text { MP2/G3Large } & & \end{array}$

\section{Ethene}

$\mathrm{HF} / 6-31 \mathrm{G}(\mathrm{d}, \mathrm{p})$

$\begin{array}{rrrrr}\mathrm{H} & 1.0 & -.0000000042 & .9153882646 & 1.2245537659 \\ \mathrm{C} & 6.0 & .0000000007 & -.0000003683 & .6582259521 \\ \mathrm{C} & 6.0 & -.0000000081 & .0000003661 & -.6582259527 \\ \mathrm{H} & 1.0 & .0000000056 & -.9153880462 & 1.2245537385 \\ \mathrm{H} & 1.0 & .0000000080 & .9153880469 & -1.2245537389 \\ \mathrm{H} & 1.0 & -.0000000019 & -.9153882631 & -1.2245537649\end{array}$

Energy

$\mathrm{ZPE}$

$\operatorname{MP} 2 / 6-31 G(d, p)$

$\begin{array}{rrrrr}\mathrm{H} & 1.0 & -.0000000059 & .9208074722 & 1.2333218197 \\ \mathrm{C} & 6.0 & .0000000091 & -.0000210249 & .6676398419 \\ \mathrm{C} & 6.0 & -.0000000199 & .0000209377 & -.6676398465 \\ \mathrm{H} & 1.0 & .0000000039 & -.9207941911 & 1.2333192656 \\ \mathrm{H} & 1.0 & .0000000114 & .9207942319 & -1.2333192883 \\ \mathrm{H} & 1.0 & .0000000015 & -.9208074259 & -1.2333217925\end{array}$

Energy

$\mathrm{ZPE}$

MP2 / 6-311+G (3df, 2p)
-78.0388414542 Hartree

0.053396 ARTREE/MOLECULE

-78.3172820048 Hartree .052343 HARTREE/MOLECULE

-78.4049919373 Hartree 


\section{Ethane}

$\operatorname{MP} 2 / 6-31 G(d, p)$

$\begin{array}{rrrrr}\mathrm{C} & 6.0 & -0.0000001010 & -0.0000008758 & -0.4372013274 \\ \mathrm{C} & 6.0 & -0.0000003626 & 0.0000007552 & 1.0862013968 \\ \mathrm{H} & 1.0 & 0.5076613493 & 0.8792949809 & 1.4797193195 \\ \mathrm{H} & 1.0 & 0.5076615159 & -0.8792958650 & 1.4797190496 \\ \mathrm{H} & 1.0 & -1.0153226998 & -0.0000002911 & 1.4797189528 \\ \mathrm{H} & 1.0 & 1.0153226458 & 0.0000001559 & -0.8307192290 \\ \mathrm{H} & 1.0 & -0.5076617146 & 0.8792956601 & -0.8307189438 \\ \mathrm{H} & 1.0 & -0.5076615271 & -0.8792950362 & -0.8307189515\end{array}$

Energy

-79.5434005239 Hartree

$\mathrm{ZPE}$

MP2 / 6-311+G (3df, 2p)

0.077460 HARTREE/MOLECULE

-79.6240909096 Hartree

\section{Methylsilane}

MP2 / 6-31G (d,p)

$\begin{array}{lrrrr}\mathrm{H} & 1.0 & 0.0179145987 & 0.0310297890 & -0.0015281156 \\ \mathrm{C} & 6.0 & 0.0047770721 & 0.0081610536 & 1.0866534269 \\ \mathrm{H} & 1.0 & 1.0350461448 & 0.0310300902 & 1.4369466968 \\ \mathrm{SI} & 14.0 & -0.8835255156 & -1.5303006628 & 1.7147436079 \\ \mathrm{H} & 1.0 & -2.2835052532 & -1.5544948048 & 1.2345901132 \\ \mathrm{H} & 1.0 & -0.2044792179 & -2.7548202017 & 1.2345863400 \\ \mathrm{H} & 1.0 & -0.8974867731 & -1.5544958526 & 3.1947125261 \\ \mathrm{H} & 1.0 & -0.4906820677 & 0.9119439621 & 1.4369605396 \\ & & & & \\ \text { Energy } & & & -330.5336325327 & \text { Hartree } \\ \text { ZPE } & & -330.6314127365 & \text { Hartree } \\ \text { MP2/6-311+G(3df,2p) } & \end{array}$

Previously Reported compounds: ethyl methyl sulfoxide, methyl vinyl sulfoxide, and their respective transition states and products. See J. Org. Chem. 2001, 66, 8722-8736. 
Table S2. Calculated heats of isodesmic reactions $\left(\mathrm{kcal} \mathrm{mol}^{-1}\right)$.

Reaction

$\Delta \mathrm{H}$

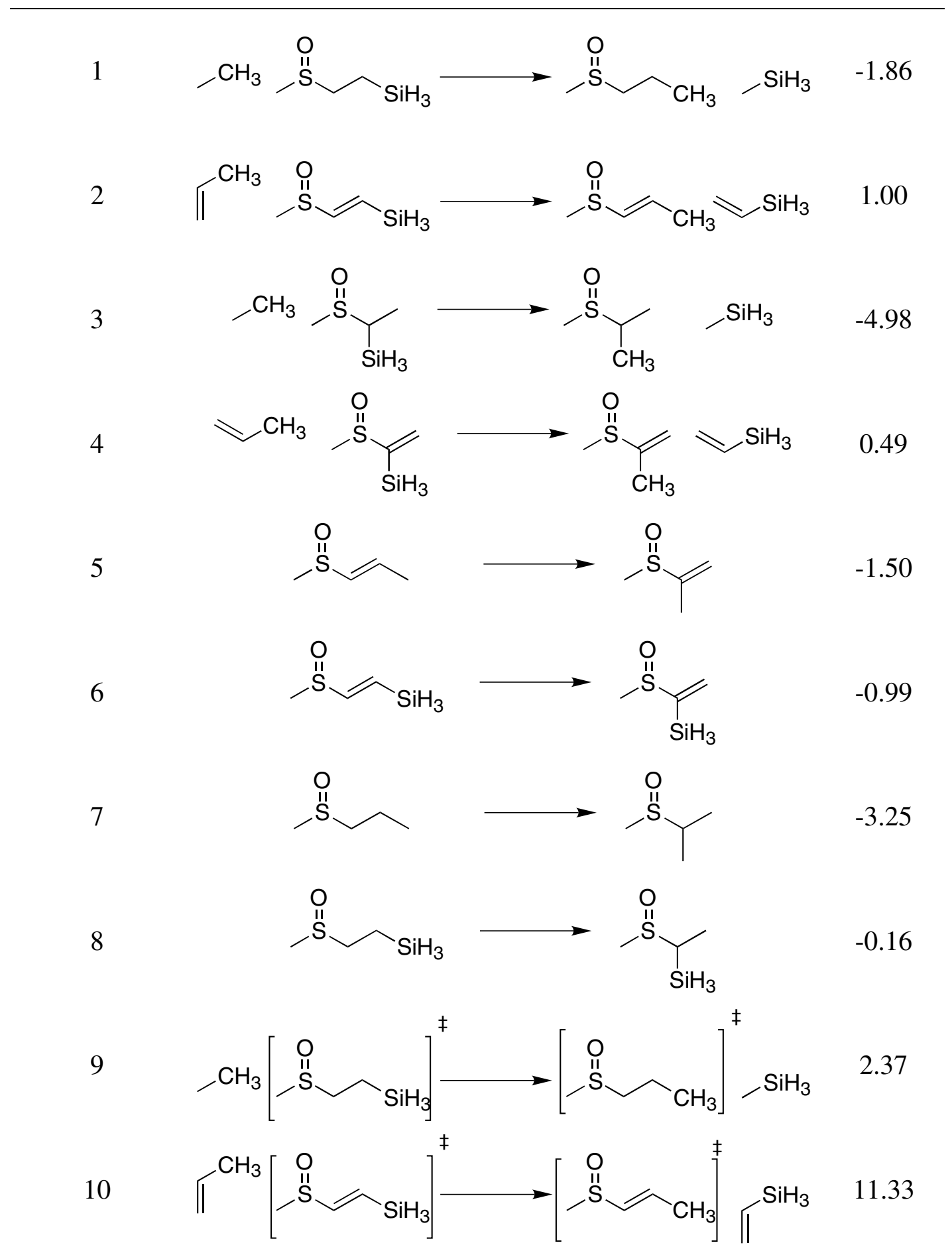


11

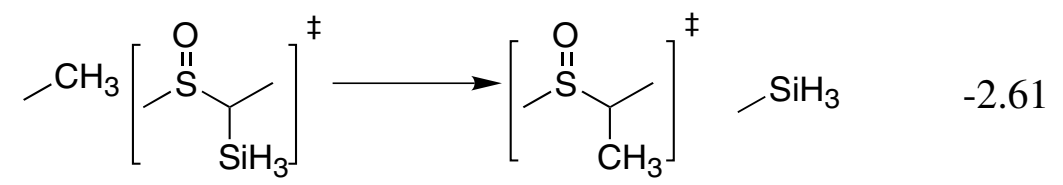

12

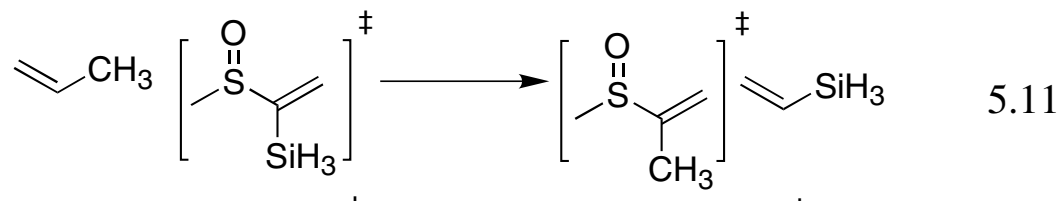

13

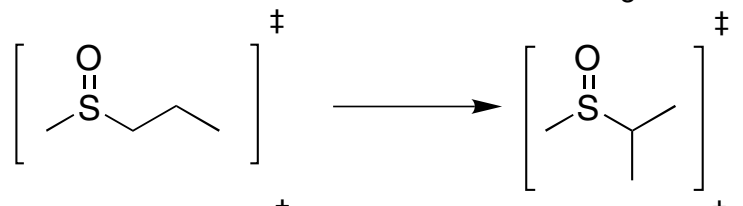

14

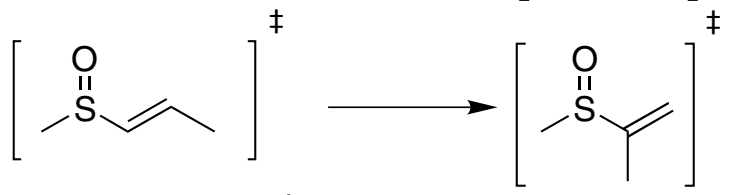

15

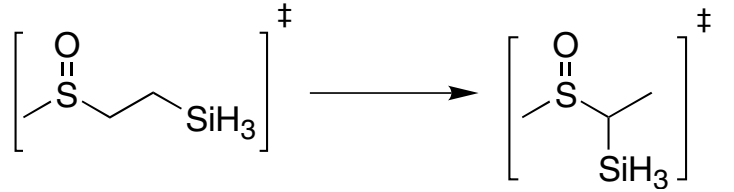

16

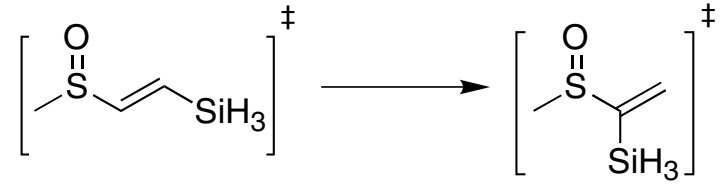

17<smiles>C#[SH]CCCC#CC</smiles>

18

$\neg \mathrm{SiH}_{3}-\mathrm{CH}_{3} \longrightarrow \widehat{C}_{\mathrm{CH}_{3}}-\mathrm{SiH}_{3}-3.93$

19

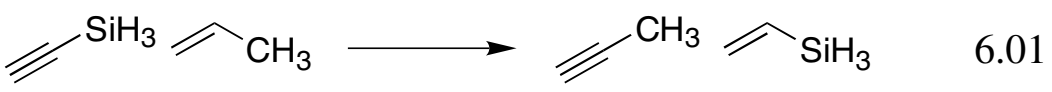


Table S3. Bond lengths, angles and orders

SM, TS and PR refer to Starting Materials (sulfoxide), Transition State, and PRoducts, respectively
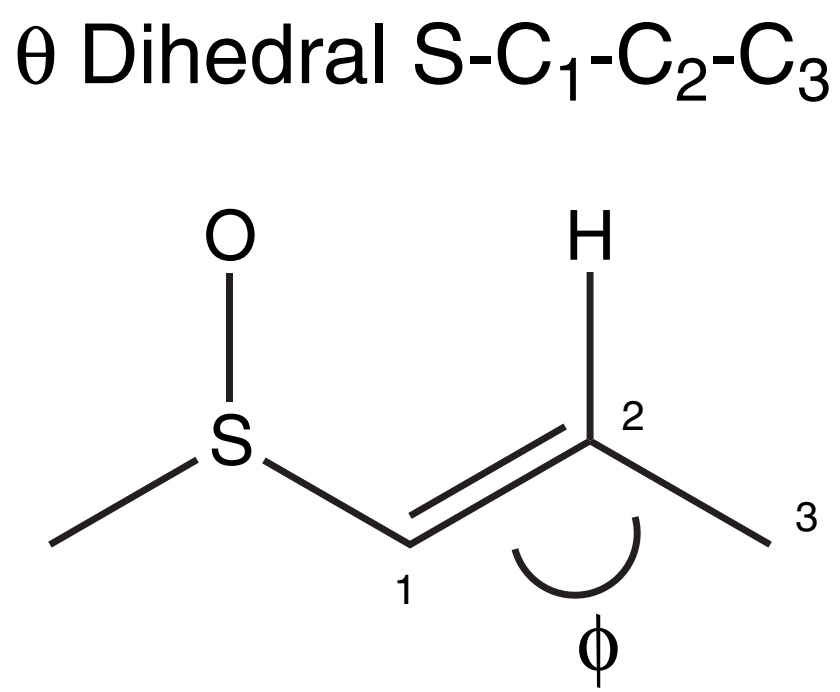

Bond lengths and Angles

\begin{tabular}{ccccccccc}
\hline & $\mathrm{S}-\mathrm{O}$ & $\mathrm{S}-\mathrm{C}_{1}$ & $\mathrm{O}-\mathrm{H}$ & $\mathrm{C}_{2}-\mathrm{H}$ & $\mathrm{C}_{1}-\mathrm{C}_{2}$ & $\mathrm{C}_{3}-\mathrm{C}_{2}$ & $\theta$ & $\phi$ \\
\hline $\mathrm{SM}$ & 1.51 & 1.79 & -- & 1.09 & 1.33 & 1.50 & -177.543 & 124.679 \\
$\mathrm{TS}$ & 1.59 & 2.30 & 1.18 & 1.41 & 1.26 & 1.49 & 178.065 & 148.202 \\
$\mathrm{PR}$ & 1.69 & -- & 0.97 & -- & 1.22 & 1.46 & -- & 180.000 \\
\hline
\end{tabular}

Bond Orders

\begin{tabular}{ccccccc}
\hline & $\mathrm{S}-\mathrm{O}$ & $\mathrm{S}-\mathrm{C}_{1}$ & $\mathrm{O}-\mathrm{H}$ & $\mathrm{C}_{2}-\mathrm{H}$ & $\mathrm{C}_{1}-\mathrm{C}_{2}$ & $\mathrm{C}_{3}-\mathrm{C}_{2}$ \\
\hline $\mathrm{SM}$ & 1.41 & 0.80 & -- & 0.90 & 1.78 & 0.95 \\
$\mathrm{TS}$ & 1.06 & 0.40 & 0.45 & 0.39 & 2.24 & 0.93 \\
PR & 0.82 & -- & 0.83 & -- & 2.76 & 0.95 \\
\hline
\end{tabular}

Mulliken Charges

\begin{tabular}{ccccccc}
\hline & $\mathrm{S}$ & $\mathrm{O}$ & $\mathrm{H}$ & $\mathrm{C}_{1}$ & $\mathrm{C}_{2}$ & $\mathrm{C}_{3}$ \\
\hline $\mathrm{SM}$ & 0.83 & -0.66 & 0.16 & -0.36 & -0.06 & -0.37 \\
$\mathrm{TS}$ & 0.55 & -0.62 & 0.29 & -0.28 & -0.10 & -0.36 \\
$\mathrm{PR}$ & 0.33 & -0.62 & 0.35 & -0.36 & 0.12 & -0.41 \\
\hline
\end{tabular}




\section{$\theta$ Dihedral $S-\mathrm{C}_{1}-\mathrm{C}_{2}-\mathrm{Si}$}

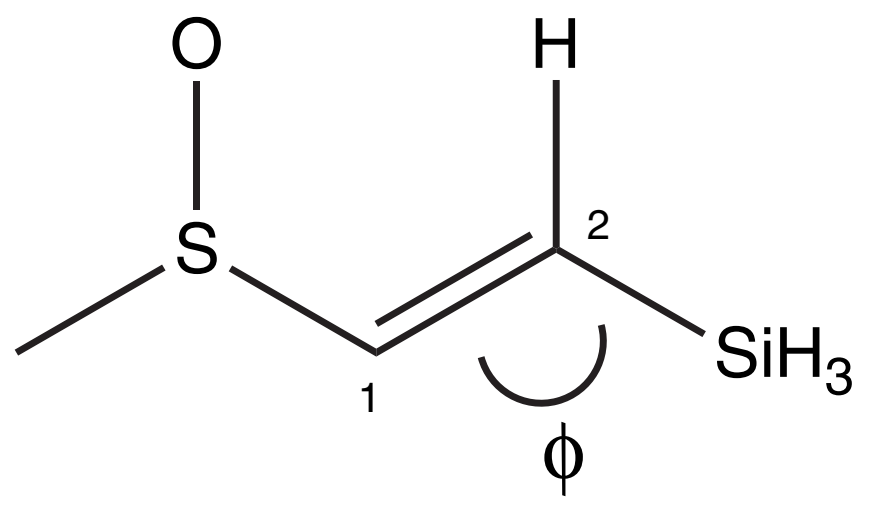

Bond lengths and Angles

\begin{tabular}{|c|c|c|c|c|c|c|c|c|}
\hline & $\mathrm{S}-\mathrm{O}$ & S-C $C_{1}$ & $\mathrm{O}-\mathrm{H}$ & $\mathrm{C}_{2}-\mathrm{H}$ & $\mathrm{C}_{1}-\mathrm{C}_{2}$ & $\mathrm{Si}-\mathrm{C}_{2}$ & $\theta$ & $\phi$ \\
\hline SM & 1.51 & 1.80 & -- & 1.09 & 1.34 & 1.87 & -178.088 & 122.542 \\
\hline $\mathrm{TS}$ & 1.59 & 2.24 & 1.16 & 1.43 & 1.27 & 1.84 & 178.385 & 145.220 \\
\hline PR & 1.69 & -- & 0.97 & -- & 1.22 & 1.83 & -- & 179.811 \\
\hline \multicolumn{9}{|c|}{ Bond Orders } \\
\hline & $\mathrm{S}-\mathrm{O}$ & S-C $\mathrm{C}_{1}$ & $\mathrm{O}-\mathrm{H}$ & $\mathrm{C}_{2}-\mathrm{H}$ & $\mathrm{C}_{1}-\mathrm{C}_{2}$ & $\mathrm{Si}-\mathrm{C}_{2}$ & & \\
\hline SM & 1.42 & 0.78 & -- & 0.88 & 1.76 & 0.92 & & \\
\hline TS & 1.06 & 0.43 & 0.45 & 0.38 & 2.11 & 0.93 & & \\
\hline PR & 0.82 & -- & 0.83 & -- & 2.52 & 1.00 & & \\
\hline \multicolumn{9}{|c|}{ Mulliken Charges } \\
\hline & & $S$ & $\mathrm{O}$ & $\mathrm{H}$ & $\mathrm{C}_{1}$ & $\mathrm{C}_{2}$ & \multicolumn{2}{|l|}{$\mathrm{Si}$} \\
\hline & SM & 0.83 & -0.65 & 0.17 & -0.33 & -0.25 & \multicolumn{2}{|l|}{0.48} \\
\hline & $\mathrm{TS}$ & 0.58 & -0.63 & 0.30 & -0.23 & -0.29 & \multicolumn{2}{|l|}{0.46} \\
\hline & PR & 0.33 & -0.62 & 0.35 & -0.11 & -0.19 & \multicolumn{2}{|l|}{0.41} \\
\hline
\end{tabular}




\section{$\theta$ Dihedral Si- $\mathrm{C}_{1}-\mathrm{C}_{2}-\mathrm{H}$}

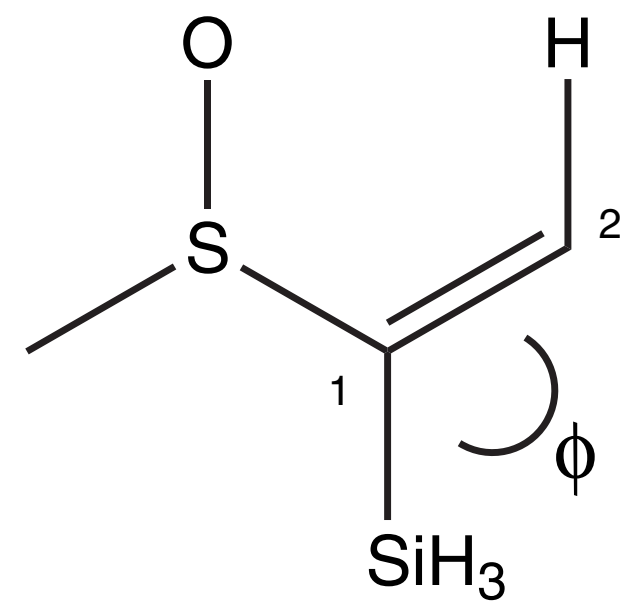

Bond lengths and Angles

\begin{tabular}{ccccccccc}
\hline & $\mathrm{S}-\mathrm{O}$ & $\mathrm{S}^{-} \mathrm{C}_{1}$ & $\mathrm{O}-\mathrm{H}$ & $\mathrm{C}_{2}-\mathrm{H}$ & $\mathrm{C}_{1}-\mathrm{C}_{2}$ & $\mathrm{Si}-\mathrm{C}_{1}$ & $\theta$ & $\phi$ \\
\hline $\mathrm{SM}$ & 1.51 & 1.80 & -- & 1.08 & 1.34 & 1.88 & 175.616 & 123.101 \\
$\mathrm{TS}$ & 1.58 & 2.39 & 1.21 & 1.35 & 1.27 & 1.84 & 173.353 & 155.394 \\
$\mathrm{PR}$ & 1.69 & -- & 0.97 & -- & 1.22 & 1.83 & -- & 179.811 \\
\hline
\end{tabular}

Bond Orders

\begin{tabular}{ccccccc}
\hline & $\mathrm{S}-\mathrm{O}$ & $\mathrm{S}^{-\mathrm{C}_{1}}$ & $\mathrm{O}-\mathrm{H}$ & $\mathrm{C}_{2}-\mathrm{H}$ & $\mathrm{C}_{1}-\mathrm{C}_{2}$ & $\mathrm{Si}^{-} \mathrm{C}_{1}$ \\
\hline $\mathrm{SM}$ & 1.41 & 0.76 & -- & 0.90 & 1.75 & 0.89 \\
$\mathrm{TS}$ & 1.08 & 0.37 & 0.41 & 0.42 & 2.13 & 0.95 \\
$\mathrm{PR}$ & 0.82 & -- & 0.83 & -- & 2.52 & 1.00 \\
\hline
\end{tabular}

Mulliken Charges

\begin{tabular}{ccccccc}
\hline & $\mathrm{S}$ & $\mathrm{O}$ & $\mathrm{H}$ & $\mathrm{C}_{1}$ & $\mathrm{C}_{2}$ & $\mathrm{Si}$ \\
\hline $\mathrm{SM}$ & 0.84 & -0.65 & 0.17 & -0.37 & -0.27 & 0.48 \\
$\mathrm{TS}$ & 0.55 & -0.60 & 0.29 & -0.19 & -0.31 & 0.46 \\
$\mathrm{PR}$ & 0.33 & -0.62 & 0.35 & -0.19 & -0.11 & 0.41 \\
\hline
\end{tabular}




\section{$\theta$ Dihedral $\mathrm{C}_{3}-\mathrm{C}_{1}-\mathrm{C}_{2}-\mathrm{H}$}

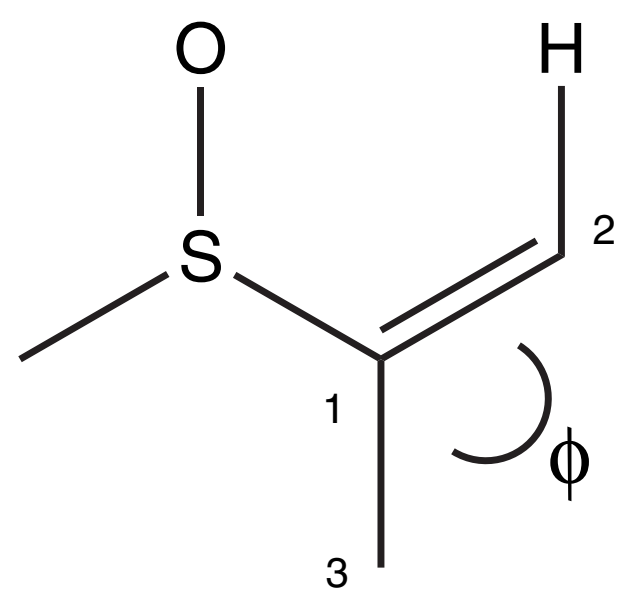

Bond lengths and Angles

\begin{tabular}{|c|c|c|c|c|c|c|c|c|}
\hline & $\mathrm{S}-\mathrm{O}$ & $\mathrm{S}-\mathrm{C}_{1}$ & $\mathrm{O}-\mathrm{H}$ & $\mathrm{C}_{2}-\mathrm{H}$ & $\mathrm{C}_{1}-\mathrm{C}_{2}$ & $\mathrm{C}_{3}-\mathrm{C}_{1}$ & $\theta$ & $\phi$ \\
\hline SM & 1.51 & 1.81 & -- & 1.08 & 1.33 & 1.50 & 178.686 & 126.626 \\
\hline $\mathrm{TS}$ & 1.59 & 2.32 & 1.17 & 1.41 & 1.26 & 1.48 & -0.712 & 22.524 \\
\hline PR & 1.69 & -- & 0.97 & -- & 1.22 & 1.46 & -- & 180.000 \\
\hline \multicolumn{9}{|c|}{ Bond Orders } \\
\hline & $\mathrm{S}-\mathrm{O}$ & $\mathrm{S}-\mathrm{C}_{1}$ & $\mathrm{O}-\mathrm{H}$ & $\mathrm{C}_{2}-\mathrm{H}$ & $\mathrm{C}_{1}-\mathrm{C}_{2}$ & $\mathrm{C}_{3}-\mathrm{C}_{1}$ & & \\
\hline SM & 1.41 & 0.78 & -- & 0.90 & 1.78 & 0.92 & & \\
\hline $\mathrm{TS}$ & 1.06 & 0.39 & 0.46 & 0.37 & 2.25 & 0.94 & & \\
\hline PR & 0.82 & -- & 0.83 & -- & 2.76 & 0.95 & & \\
\hline
\end{tabular}

Mulliken Charges

\begin{tabular}{ccccccc}
\hline & $\mathrm{S}$ & $\mathrm{O}$ & $\mathrm{H}$ & $\mathrm{C}_{1}$ & $\mathrm{C}_{2}$ & $\mathrm{C}_{3}$ \\
\hline $\mathrm{SM}$ & 0.83 & -0.66 & 0.16 & -0.18 & -0.25 & -0.37 \\
$\mathrm{TS}$ & 0.53 & -0.62 & 0.30 & 0.00 & -0.36 & -0.38 \\
$\mathrm{PR}$ & 0.33 & -0.62 & 0.35 & 0.13 & -0.36 & -0.40 \\
\hline
\end{tabular}




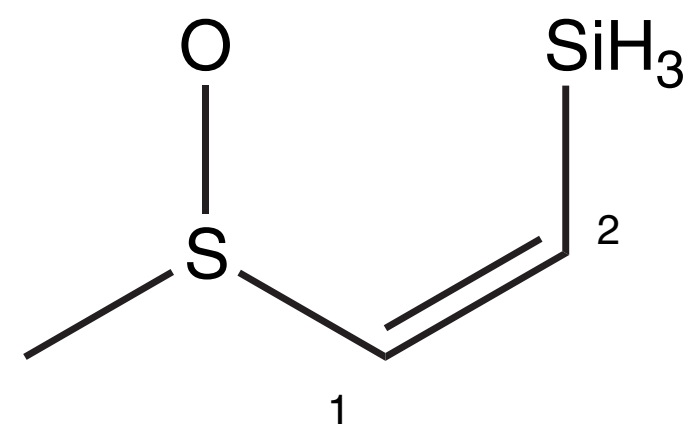

Elimination by attack at $\mathrm{Si}$

Bond lengths and Angles

\begin{tabular}{|c|c|c|c|c|c|}
\hline & $\mathrm{S}-\mathrm{O}$ & $\mathrm{S}-\mathrm{C}_{1}$ & $\mathrm{O}-\mathrm{Si}$ & $\mathrm{C}_{1}-\mathrm{C}_{2}$ & $\mathrm{Si}_{-} \mathrm{C}_{2}$ \\
\hline SM & 1.51 & 1.79 & -- & 1.34 & 1.88 \\
\hline $\mathrm{TS}$ & 1.61 & 2.29 & 1.78 & 1.25 & 2.36 \\
\hline PR & 1.70 & 1.79 & 1.69 & 1.22 & -- \\
\hline \multicolumn{6}{|c|}{ Bond Orders } \\
\hline & $\mathrm{S}-\mathrm{O}$ & $\mathrm{S}-\mathrm{C}_{1}$ & $\mathrm{O}-\mathrm{Si}$ & $\mathrm{C}_{1}-\mathrm{C}_{2}$ & $\mathrm{Si}-\mathrm{C}_{2}$ \\
\hline SM & 1.42 & 0.80 & -- & 1.73 & 0.92 \\
\hline $\mathrm{TS}$ & 0.88 & 0.37 & 0.70 & 2.38 & 0.28 \\
\hline PR & 0.76 & -- & 0.90 & 2.84 & -- \\
\hline \multicolumn{6}{|c|}{ Mulliken Charges } \\
\hline & S & $\mathrm{O}$ & $\mathrm{Si}$ & $\mathrm{C}_{1}$ & $\mathrm{C}_{2}$ \\
\hline SM & 0.83 & -0.66 & 0.48 & -0.31 & -0.27 \\
\hline $\mathrm{TS}$ & 0.58 & -0.66 & 0.66 & -0.19 & -0.31 \\
\hline PR & 0.36 & -0.67 & 0.72 & -0.19 & -0.19 \\
\hline
\end{tabular}




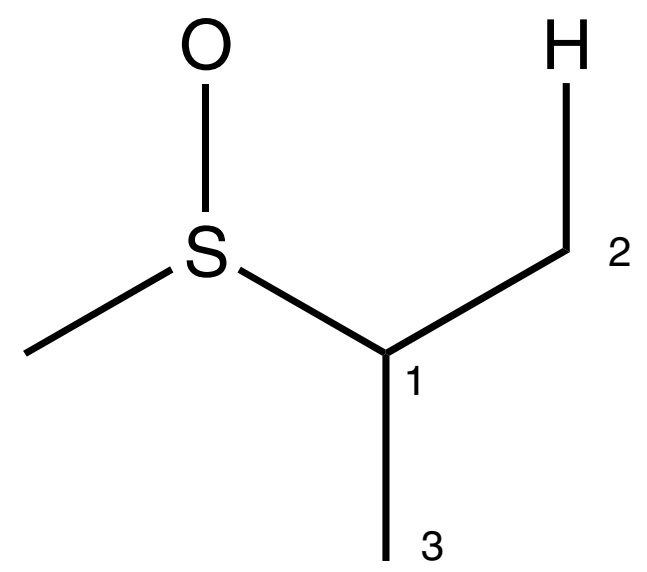

Bond lengths and Angles

\begin{tabular}{cccccc}
\hline & $\mathrm{S}-\mathrm{O}$ & $\mathrm{S}^{-} \mathrm{C}_{1}$ & $\mathrm{O}-\mathrm{H}$ & $\mathrm{H}-\mathrm{C}_{2}$ & $\mathrm{C}_{1}-\mathrm{C}_{2}$ \\
\hline $\mathrm{SM}$ & 1.52 & 1.836 & -- & 1.09 & 1.52 \\
$\mathrm{TS}$ & 1.59 & 2.35 & 1.21 & 1.38 & 1.40 \\
$\mathrm{PR}$ & 1.69 & -- & 0.97 & -- & 1.34 \\
\hline
\end{tabular}

Bond Orders

\begin{tabular}{cccccc}
\hline & $\mathrm{S}-\mathrm{O}$ & $\mathrm{S}^{-\mathrm{C}_{1}}$ & $\mathrm{O}-\mathrm{H}$ & $\mathrm{H}-\mathrm{C}_{2}$ & $\mathrm{C}_{1}-\mathrm{C}_{2}$ \\
\hline $\mathrm{SM}$ & 1.38 & 0.77 & -- & 0.91 & 0.94 \\
$\mathrm{TS}$ & 1.07 & 0.42 & 0.42 & 0.42 & 1.29 \\
$\mathrm{PR}$ & 0.82 & -- & 0.83 & -- & 1.83 \\
\hline
\end{tabular}

Mulliken Charges

\begin{tabular}{ccccccc}
\hline & $\mathrm{S}$ & $\mathrm{O}$ & $\mathrm{H}$ & $\mathrm{C}_{1}$ & $\mathrm{C}_{2}$ & $\mathrm{C}_{3}$ \\
\hline $\mathrm{SM}$ & 0.83 & -0.67 & 0.16 & -0.30 & -0.35 & -0.35 \\
$\mathrm{TS}$ & 0.55 & -0.62 & 0.30 & -0.16 & -0.42 & -0.33 \\
$\mathrm{PR}$ & 0.32 & -0.62 & 0.35 & -0.05 & -0.25 & -0.37 \\
\hline
\end{tabular}




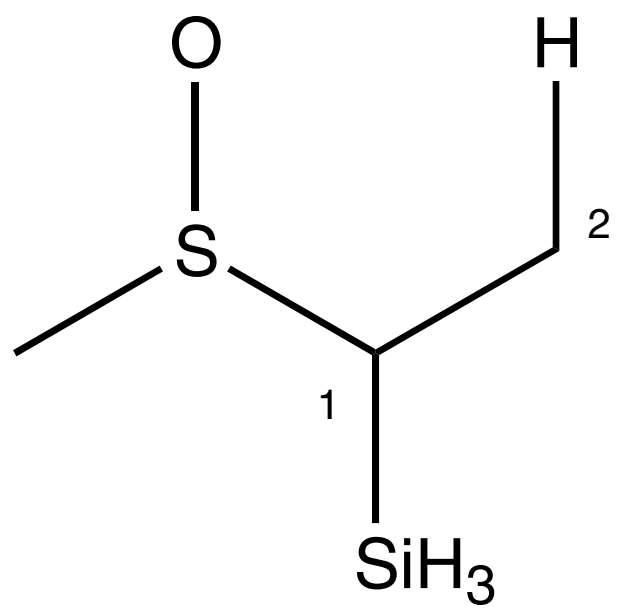

Bond lengths and Angles

\begin{tabular}{ccccccc}
\hline & $\mathrm{S}-\mathrm{O}$ & $\mathrm{S}-\mathrm{C}_{1}$ & $\mathrm{O}-\mathrm{H}$ & $\mathrm{H}-\mathrm{C}_{2}$ & $\mathrm{C}_{1}-\mathrm{C}_{2}$ & $\mathrm{C}_{1}-\mathrm{Si}$ \\
\hline $\mathrm{SM}$ & 1.52 & 1.82 & -- & 1.09 & 1.53 & 1.91 \\
$\mathrm{TS}$ & 1.58 & 2.40 & 1.23 & 1.35 & 1.41 & 1.87 \\
$\mathrm{PR}$ & 1.69 & -- & 0.97 & -- & 1.34 & 1.87 \\
\hline
\end{tabular}

\begin{tabular}{ccccccc}
\hline \multicolumn{7}{c}{ Bond Orders } \\
& $\mathrm{S}-\mathrm{O}$ & $\mathrm{S}-\mathrm{C}_{1}$ & $\mathrm{O}-\mathrm{H}$ & $\mathrm{H}-\mathrm{C}_{2}$ & $\mathrm{C}_{1}-\mathrm{C}_{2}$ & $\mathrm{C}_{1}-\mathrm{Si}$ \\
\hline $\mathrm{SM}$ & 1.34 & 0.79 & -- & 0.91 & 0.92 & 0.86 \\
$\mathrm{TS}$ & 1.09 & 0.40 & 0.39 & 0.45 & 1.27 & 0.92 \\
$\mathrm{PR}$ & 0.82 & -- & 0.83 & -- & 1.80 & 0.94 \\
\hline
\end{tabular}

Mulliken Charges

\begin{tabular}{cccccccc}
\hline & $\mathrm{S}$ & $\mathrm{O}$ & $\mathrm{H}$ & $\mathrm{C}_{1}$ & $\mathrm{C}_{2}$ & $\mathrm{Si}$ & $\mathrm{Si} \underline{\mathrm{H}}$ \\
\hline $\mathrm{SM}$ & 0.84 & -0.67 & 0.16 & -0.50 & -0.36 & 0.51 & -0.10 \\
$\mathrm{TS}$ & 0.56 & -0.61 & 0.29 & -0.35 & -0.41 & 0.50 & -0.11 \\
$\mathrm{PR}$ & 0.32 & -0.62 & 0.35 & -0.25 & -0.23 & 0.47 & -0.12 \\
\hline
\end{tabular}




\section{$\theta$ Dihedral S- $\mathrm{C}_{1}-\mathrm{C}_{2}-\mathrm{Si}$}

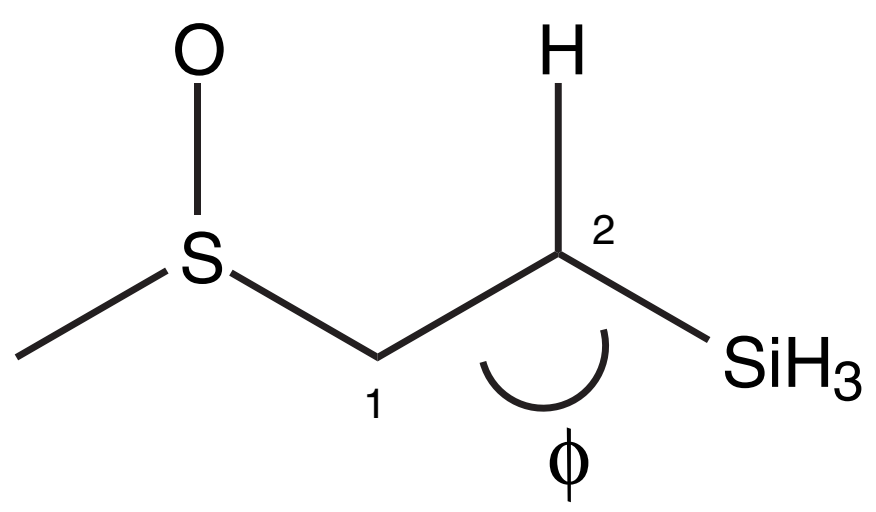

Bond lengths and Angles

\begin{tabular}{ccccccccc}
\hline & $\mathrm{S}-\mathrm{O}$ & $\mathrm{S}-\mathrm{C}_{1}$ & $\mathrm{O}-\mathrm{H}$ & $\mathrm{C}_{2}-\mathrm{H}$ & $\mathrm{C}_{1}-\mathrm{C}_{2}$ & $\mathrm{Si}-\mathrm{C}_{2}$ & $\theta$ & $\phi$ \\
$\mathrm{SM}$ & 1.51 & 1.83 & -- & 1.09 & 1.52 & 1.89 & 166.01 & 112.720 \\
$\mathrm{TS}$ & 1.59 & 2.29 & 1.20 & 1.40 & 1.41 & 1.86 & 112.72 & 119.059 \\
$\mathrm{PR}$ & 1.69 & -- & 0.97 & -- & 1.34 & 1.87 & -- & 122.404 \\
\hline & & & & & & & & \\
\hline $\mathrm{SM}$ & 1.39 & 0.78 & -- & 0.89 & 0.92 & 0.90 & & \\
\hline $\mathrm{TS}$ & 1.06 & 0.45 & 0.41 & 0.43 & 1.26 & 0.91 & & \\
$\mathrm{PR}$ & 0.82 & -- & 0.83 & -- & 1.80 & 0.94 & \\
\hline
\end{tabular}

Mulliken Charges

\begin{tabular}{ccccccc}
\hline & $\mathrm{S}$ & $\mathrm{O}$ & $\mathrm{H}$ & $\mathrm{C}_{1}$ & $\mathrm{C}_{2}$ & $\mathrm{Si}$ \\
\hline $\mathrm{SM}$ & 0.83 & -0.67 & 0.17 & -0.43 & -0.42 & 0.51 \\
$\mathrm{TS}$ & 0.58 & -0.63 & 0.30 & -0.31 & -0.45 & 0.50 \\
$\mathrm{PR}$ & 0.33 & -0.62 & 0.35 & -0.23 & -0.25 & 0.47 \\
\hline
\end{tabular}




\section{$\theta$ Dihedral $S-\mathrm{C}_{1}-\mathrm{C}_{2}-\mathrm{Si}$}

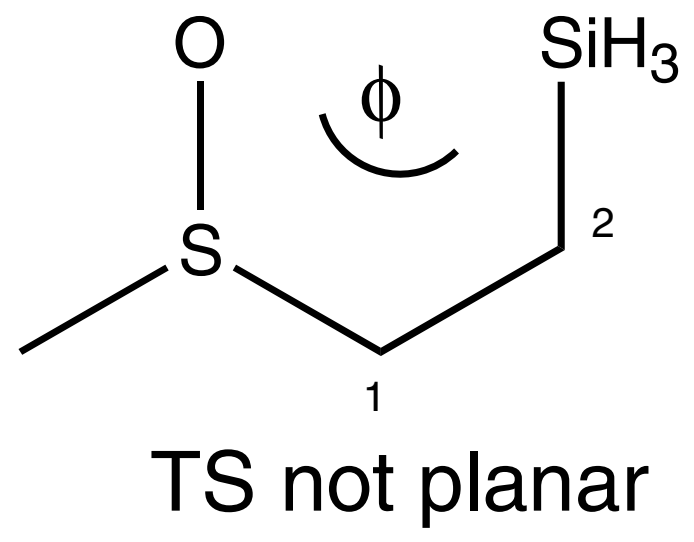

Attack at Si

Bond lengths and Angles

\begin{tabular}{|c|c|c|c|c|c|c|}
\hline & $\mathrm{S}-\mathrm{O}$ & $\mathrm{S}-\mathrm{C}_{1}$ & $\mathrm{O}-\mathrm{Si}$ & $\mathrm{C}_{1}-\mathrm{C}_{2}$ & $\mathrm{Si}-\mathrm{C}_{2}$ & $\theta$ \\
\hline SM & 1.51 & 1.83 & -- & 1.52 & 1.89 & 166.01 \\
\hline $\mathrm{TS}$ & 1.60 & 2.28 & 1.83 & 1.39 & 2.33 & -9.48 \\
\hline \multirow[t]{11}{*}{ PR } & 1.70 & 1.79 & 1.69 & 1.34 & -- & \\
\hline & \multicolumn{6}{|c|}{ Bond Orders } \\
\hline & & $\mathrm{S}-\mathrm{O}$ & $\mathrm{S}-\mathrm{C}_{1}$ & $\mathrm{O}-\mathrm{Si}$ & $\mathrm{C}_{1}-\mathrm{C}_{2}$ & $\mathrm{Si}-\mathrm{C}_{2}$ \\
\hline & $\mathrm{SM}$ & 1.39 & 0.78 & -- & 0.92 & 0.90 \\
\hline & $\mathrm{TS}$ & 0.92 & 0.40 & 0.40 & 1.33 & 0.37 \\
\hline & PR & 0.76 & -- & 0.90 & 1.80 & -- \\
\hline & \multicolumn{6}{|c|}{ Mulliken Charges } \\
\hline & & S & $\mathrm{O}$ & $\mathrm{C}_{1}$ & $\mathrm{C}_{2}$ & $\mathrm{Si}$ \\
\hline & $\mathrm{SM}$ & 0.83 & -0.67 & -0.43 & -0.42 & 0.51 \\
\hline & $\mathrm{TS}$ & 0.61 & -0.65 & -0.29 & -0.41 & 0.63 \\
\hline & PR & 0.36 & -0.67 & -0.23 & -0.25 & 0.72 \\
\hline
\end{tabular}




\section{$\theta$ Dihedral $\mathrm{S}-\mathrm{C}_{1}-\mathrm{C}_{2}-\mathrm{C}_{3}$}

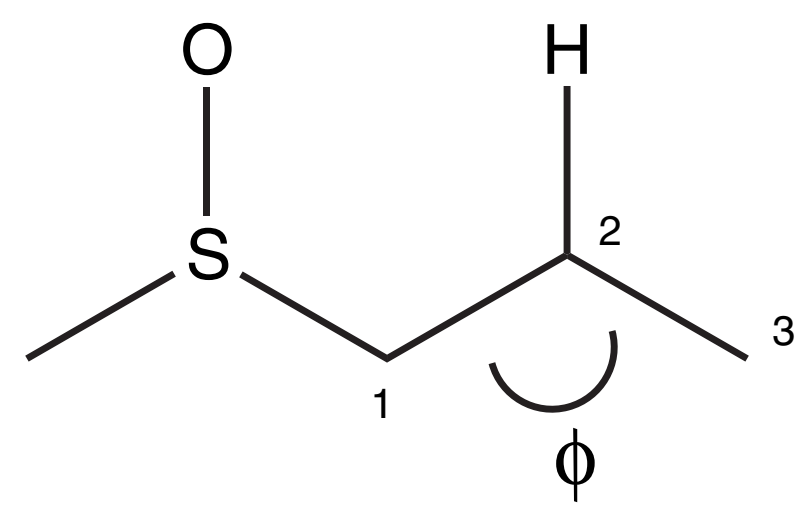

trans-TS_propyl methyl sulfoxide elimination

Bond lengths and Angles

\begin{tabular}{ccccccccc}
\hline & $\mathrm{S}-\mathrm{O}$ & $\mathrm{S}-\mathrm{C}_{1}$ & $\mathrm{O}-\mathrm{H}$ & $\mathrm{C}_{2}-\mathrm{H}$ & $\mathrm{C}_{1}-\mathrm{C}_{2}$ & $\mathrm{C}_{3}-\mathrm{C}_{2}$ & $\theta$ & $\phi$ \\
\hline $\mathrm{SM}$ & 1.51 & 1.82 & -- & 1.09 & 1.52 & 1.52 & 165.684 & 111.805 \\
$\mathrm{TS}$ & 1.58 & 2.35 & 1.23 & 1.35 & 1.41 & 1.52 & 107.722 & 119.308 \\
$\mathrm{PR}$ & 1.69 & -- & 0.97 & -- & 1.34 & 1.50 & -- & 124.454 \\
\hline $\mathrm{SM}$ & 1.40 & 0.79 & -- & 0.91 & 0.91 & 0.93 & & \\
\hline $\mathrm{TS}$ & 1.08 & 0.42 & 0.40 & 0.44 & 1.28 & 0.93 & & \\
\hline $\mathrm{PR}$ & 0.82 & -- & 0.83 & -- & 1.83 & 0.95 & & \\
\hline
\end{tabular}

Mulliken Charges

\begin{tabular}{ccccccc}
\hline & $\mathrm{S}$ & $\mathrm{O}$ & $\mathrm{H}$ & $\mathrm{C}_{1}$ & $\mathrm{C}_{2}$ & $\mathrm{C}_{3}$ \\
\hline $\mathrm{SM}$ & 0.83 & -0.67 & 0.16 & -0.43 & -0.22 & -0.33 \\
$\mathrm{TS}$ & 0.55 & -0.62 & 0.29 & -0.30 & -0.28 & -0.32 \\
$\mathrm{PR}$ & 0.33 & -0.62 & 0.35 & -0.25 & -0.06 & -0.33 \\
\hline
\end{tabular}


